\title{
RISING FROM THE ASHES: MID-INFRARED RE-BRIGHTENING OF THE IMPOSTOR SN 2010da IN NGC 300
}

\author{
Ryan M. Lau ${ }^{1,2}$, Mansi M. Kasliwal ${ }^{2}$, Howard E. Bond ${ }^{3,4}$, Nathan Smith ${ }^{5}$, Ori D. Fox ${ }^{4}$, Robert Carlon $^{6}$, \\ ann Marie Cody $^{7}$, Carlos Contreras ${ }^{8}$, Devin Dykhoff ${ }^{6}$, Robert Gehrz ${ }^{6}$, Eric Hsiao ${ }^{9}$, Jacob Jencson $^{2}$, Rubab Khan $^{10}$, \\ Frank Masci $^{11}$, L. A. G. Monard ${ }^{12}$, Andrew J. Monson ${ }^{3}$, Nidia Morrell ${ }^{13}$, Mark Phillips ${ }^{13}$, and Michael E. Ressler ${ }^{1}$ \\ ${ }^{1}$ Jet Propulsion Laboratory, California Institute of Technology, 4800 Oak Grove Drive, Pasadena, CA 91109, USA \\ ${ }^{2}$ California Institute of Technology, Pasadena, CA 91125, USA \\ ${ }^{3}$ Department of Astronomy \& Astrophysics, Pennsylvania State University, University Park, PA 16802, USA \\ ${ }^{4}$ Space Telescope Science Institute, 3700 San Martin Drive, Baltimore, MD 21218, USA \\ ${ }^{5}$ Steward Observatory, University of Arizona, Tuscon, AZ 85721, USA \\ ${ }^{6}$ Minnesota Institute for Astrophysics, School of Physics and Astronomy, 116 Church Street, SE, \\ University of Minnesota, Minneapolis, MN 55455, USA \\ ${ }^{7}$ NASA Ames Research Center, Moffett Field, CA 94035, USA \\ ${ }^{8}$ Las Campanas Observatory, Carnegie Observatories, Casilla 601, La Serena, Chile \\ ${ }^{9}$ Department of Physics, Florida State University, 77 Chieftain Way, Tallahassee, FL 32306, USA \\ ${ }^{10}$ NASA Goddard Space Flight Center, MC 665, 8800 Greenbelt Road, Greenbelt, MD 20771, USA \\ ${ }^{11}$ Infrared Processing and Analysis Center, California Institute of Technology, M/S 100-22, Pasadena, CA 91125, USA \\ ${ }^{12}$ Bronberg and Kleinkaroo Observatories, P.O. Box 281, Calitzdorp 6660, Western Cape, South Africa \\ ${ }^{13}$ Carnegie Institution of Washington, Las Campanas Observatory, Colina el Pino, Casilla 601, La Serena, Chile \\ Received 2016 May 6; revised 2016 July 25; accepted 2016 July 26; published 2016 October 18
}

\begin{abstract}
We present multi-epoch mid-infrared (IR) photometry and the optical discovery observations of the "impostor" supernova (SN) 2010da in NGC 300 using new and archival Spitzer Space Telescope images and ground-based observatories. The mid-infrared counterpart of SN 2010da was detected as Spitzer Infrared Intensive Transient Survey (SPIRITS) 14bme in the SPIRITS, an ongoing systematic search for IR transients. Before erupting on 2010 May 24, the SN 2010da progenitor exhibited a constant mid-IR flux at 3.6 and only a slight $\sim 10 \%$ decrease at $4.5 \mu \mathrm{m}$ between 2003 November and 2007 December. A sharp increase in the $3.6 \mu \mathrm{m}$ flux followed by a rapid decrease measured $\sim 150$ days before and $\sim 80$ days after the initial outburst, respectively, reveal a mid-IR counterpart to the coincident optical and high luminosity X-ray outbursts. At late times, after the outburst ( 2000 days), the 3.6 and $4.5 \mu \mathrm{m}$ emission increased to over a factor of two times the progenitor flux and is currently observed (as of 2016 Feb) to be fading, but still above the progenitor flux. We attribute the re-brightening mid-IR emission to continued dust production and increasing luminosity of the surviving system associated with SN 2010da. We analyze the evolution of the dust temperature $\left(T_{\mathrm{d}} \sim 700-1000 \mathrm{~K}\right)$, mass $\left(M_{\mathrm{d}} \sim 0.5-3.8 \times 10^{-7} M_{\odot}\right)$, luminosity $\left(L_{\mathrm{IR}} \sim 1.3-3.5 \times 10^{4} L_{\odot}\right)$, and the equilibrium temperature radius $\left(R_{\mathrm{eq}} \sim 6.4-12.2 \mathrm{au}\right)$ in order to resolve the nature of SN 2010da. We address the leading interpretation of SN 2010da as an eruption from a luminous blue variable high-mass X-ray binary (HMXB) system. We propose that SN 2010da is instead a supergiant (sg)B[e]-HMXB based on similar luminosities and dust masses exhibited by two other known sgB[e]-HMXB systems. Additionally, the SN 2010da progenitor occupies a similar region on a mid-IR color-magnitude diagram (CMD) with known $\mathrm{sgB}$ [e] stars in the Large Magellanic Cloud. The lower limit estimated for the orbital eccentricity of the $\operatorname{sgB}[\mathrm{e}]-\mathrm{HMXB}(e>0.82)$ from X-ray luminosity measurements is high compared to known sgHMXBs and supports the claim that SN 2010da may be associated with a newly formed HMXB system.
\end{abstract}

Key words: circumstellar matter - dust, extinction - stars: evolution - stars: mass-loss - supernovae: individual (SN 2010da) - X-rays: binaries

\section{INTRODUCTION}

The transient event designated supernova (SN) 2010da was discovered as an optical transient (OT) in the nearby galaxy NGC 300 (Figure 1) on 2010 May 23 by Monard (2010). The initial classification as an SN, however, was immediately contradicted by its low outburst luminosity $\left(M_{V} \sim-10.3\right.$ at maximum; Khan et al. 2010). Mid-infrared (IR) photometry from archival Spitzer imaging data revealed that the progenitor of SN 2010da heavily obscured and shared a similar position in a mid-IR color-magnitude diagram (CMD) as luminous blue variable (LBV) candidates (Massey et al. 2007; Thompson et al. 2009; Khan et al. 2010; Laskar et al. 2010). Follow-up optical spectroscopy of SN 2010da supported the claim that it was not a true SN, but instead resembled an LBV-like outburst (Chornock \& Berger 2010; Elias-Rosa et al. 2010). Extreme outbursts from LBVs are typically associated with a class of SN impostors that are non-terminal stellar explosions with lower peak luminosities and ejecta velocities than typical Type II SNe (Smith et al. 2011; Van Dyk \& Matheson 2012).

The LBV eruption hypothesis for SN 2010da, however, is confounded by low progenitor luminosities $\left(\sim 10^{4} L_{\odot}\right.$; Prieto et al. 2010; this work) and the detection of an X-ray point source from Swift within hours of the optical detection of SN 2010da (Immler et al. 2010) that exhibited a $0.3-10 \mathrm{keV}$ luminosity of $\sim 5 \times 10^{38} \mathrm{erg} \mathrm{s}^{-1}$. Studies of the subsequent X-ray emission by Binder et al. (2016) revealed a recurring, less luminous $\left(L_{x} \sim 4 \times 10^{37} \mathrm{erg} \mathrm{s}^{-1} ; 0.35-8 \mathrm{keV}\right)$ outburst with Chandra. These X-ray luminosities are several orders of magnitude greater than the highest observed X-ray luminosities from an LBV outburst ( $\sim 10^{34} \mathrm{erg} \mathrm{s}^{-1}$, Nazé et al. 2012) and massive colliding- 


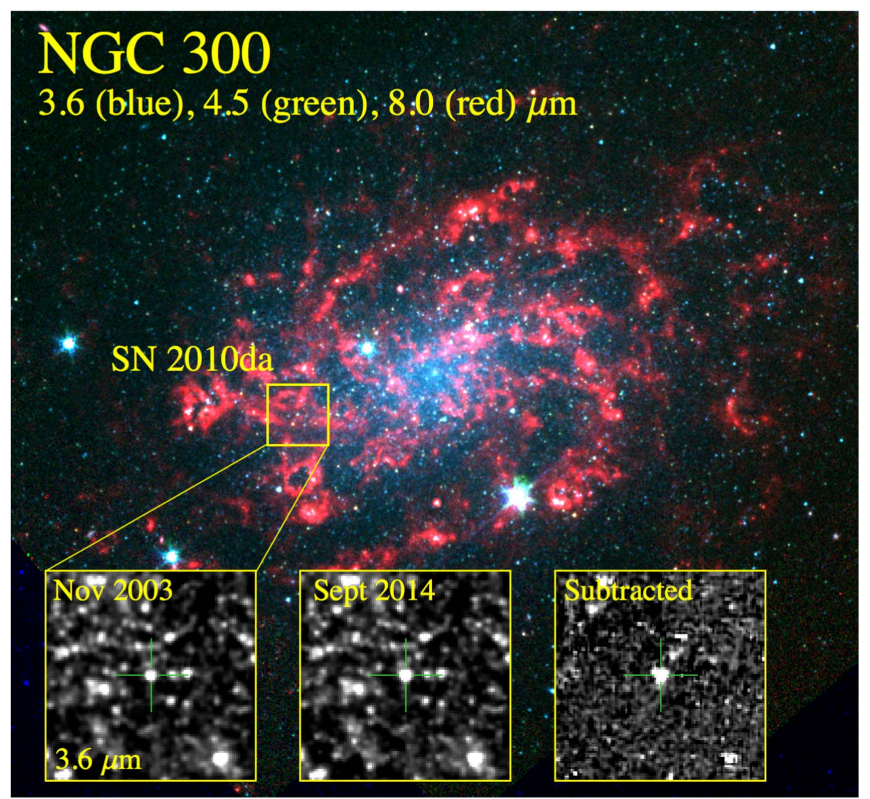

Figure 1. Spitzer/IRAC false color image of NGC 300 overlaid with the channel $1(3.6 \mu \mathrm{m})$ zoom on SN 2010da/SPIRITS 14bme taken before and after the initial optical outburst. The "Subtracted" inset shows the difference between the post-outburst and pre-outburst images.

wind binaries $\left(\sim 10^{35} \mathrm{erg} \mathrm{s}^{-1}\right.$, e.g., Guerrero \& Chu 2008). In order to reconcile the X-ray observations with the mid-IR and optical studies that implicated an LBV-like eruption, Binder et al. (2016) suggest that SN 2010da is a high-mass X-ray binary (HMXB) composed of a neutron star (NS) and an LBV-like companion. Their claim is substantiated by the presence of a young cluster in the vicinity of SN 2010da estimated to be $\lesssim 5$ Myr old based on analysis of its CMD. Binder et al. (2016) propose the interesting hypothesis that the initial outburst of SN 2010da marked the onset of the HMXB phase based on the recurring $X$-ray bursts and the presumed quiescence of the progenitor, where $L_{\mathrm{X}}<(3-9) \times 10^{36} \mathrm{erg} \mathrm{s}^{-1}$ at four epochs.

HMXBs are accretion-powered binary systems with an NS or black hole orbiting an O- or B-type companion and are subdivided into two subclasses depending on the evolutionary status of the optical companion (Liu et al. 2005; Reig 2011). The most common types of HMXBs ( 60\%; Liu et al. 2006) are known as $\mathrm{Be} / \mathrm{X}$-ray binaries (BeXBs) and host a rapidly rotating, non-supergiant B-type star with low-ionization emission lines attributed to an equatorial "excretion" disk of material ejected from the star. Variable X-ray emission from BeXBs is associated with the interactions between the circumstellar disk around the Be star and the wide, eccentric orbits of the compact object companion. The less commonly detected subclass of HMXBs host O- or B-type supergiant optical companions (sgHMXBs), where the X-ray emission is powered by accretion onto the compact companion from Roche-Lobe overflow (RLOF) and/or stellar wind capture. Wind-fed sgHMXBs exhibit persistent X-ray luminosities of $\sim 10^{35-36} \mathrm{erg} \mathrm{s}^{-1}$ while the systems that accrete from RLOF can result in higher luminosity outbursts $\left(\sim 10^{38} \mathrm{erg} \mathrm{s}^{-1}\right.$; e.g., Chaty et al. 2008).

Recent hard-X-ray observations $(\gtrsim 15 \mathrm{keV})$ less affected by extinction have revealed an emerging population of highly obscured $\left(A_{V} \gtrsim 15\right)$ sgHMXBs (e.g., Filliatre \& Chaty 2004; Chaty et al. 2008; Coleiro et al. 2013). The most intriguing are the obscured systems that host supergiant (sg)B[e]-stars, supergiant counterparts of Be stars with forbidden emission lines. $\mathrm{SgB}[\mathrm{e}]$-stars appear spectroscopically similar to LBVs and occupy similar regions of the HR diagram (e.g., Smith et al. 2004); however, they do not exhibit extreme variability in photospheric temperatures or giant eruptions associated with LBVs. Their similar spectral properties may suggest there is an evolutionary link between the two phases. Studies of such a system would be beneficial for understanding the dusty environment of highly obscured sgHMXBs. Resolving the nature of SN 2010da is therefore one of the main purposes of this paper.

In this paper, we present optical and mid-IR observations of SN 2010da and discuss the evolution of its optical and mid-IR properties (e.g., temperature and luminosity) inferred from the light curves. We address the nature of SN 2010da by proposing various interpretations (e.g., LBV eruption, merger product, HMXB) and assessing their validity given the energetics and dust properties we derive from observations. We adopt a distance of 2.0 Mpc $(\mu=26.5)$ toward NGC 300 (Dalcanton et al. 2009).

\section{OBSERVATIONS}

\subsection{Optical Discovery and Follow-up Observations}

\subsubsection{Discovery from Bronberg Observatory}

The outburst of a new OT in NGC 300 was discovered by Monard (2010) with his $0.3 \mathrm{~m}$ telescope at the Bronberg Observatory and an unfiltered ST7-XME CCD camera. The initial detection was made on 2010 May 23.16, during a morning session of $\mathrm{SN}$ searching in nearby galaxies. A confirmation image was obtained on 2010 May 24.14 with the same instrumentation. These were the first Bronberg observations of NGC 300 of the season, following its solar conjunction, showing that the eruption was already underway. The discovery light curve is shown in Figure 2 and the data are provided in Table 1. Throughout the paper, the "peak" date $\left(t_{d}=0\right)$ corresponds to the date of the optical outburst $(\mathrm{JD}=2455343.66)$.

\subsubsection{Follow-up with SMARTS}

On 2010 May 25, we began photometric monitoring of this event with the ANDICAM CCD camera on the SMARTS Consortium $^{14} 1.3 \mathrm{~m}$ telescope at Cerro Tololo Inter-American Observatory (CTIO). This monitoring continued until 2010 September 5, after which the OT became too faint for useful observations. A re-brightening of the object was detected on 2011 October 21.16 by Chornock et al. (2011). We resumed the $1.3 \mathrm{~m}$ coverage on 2011 November 12, and continued until 2011 December 20, after which the OT was again too faint for further observations.

The SMARTS monitoring was carried out by service personnel at CTIO, using BVRI filters in the Johnson-KronCousins system. The ANDICAM frames were bias-subtracted and flat-fielded in the SMARTS pipeline at Yale University. We then determined differential magnitudes between the OT and a nearby comparison star, using standard aperture-photometry tasks in IRAF. ${ }^{15}$ Absolute photometry of the comparison star (J2000: 00:55:06.47, -37:42:58.2) was determined from

\footnotetext{
14 SMARTS is the Small and Moderate Aperture Research Telescope System; http://www.astro.yale.edu/smarts.

15 IRAF is distributed by the National Optical Astronomy Observatory, which is operated by the Association of Universities for Research in Astronomy (AURA) under a cooperative agreement with the National Science Foundation.
} 


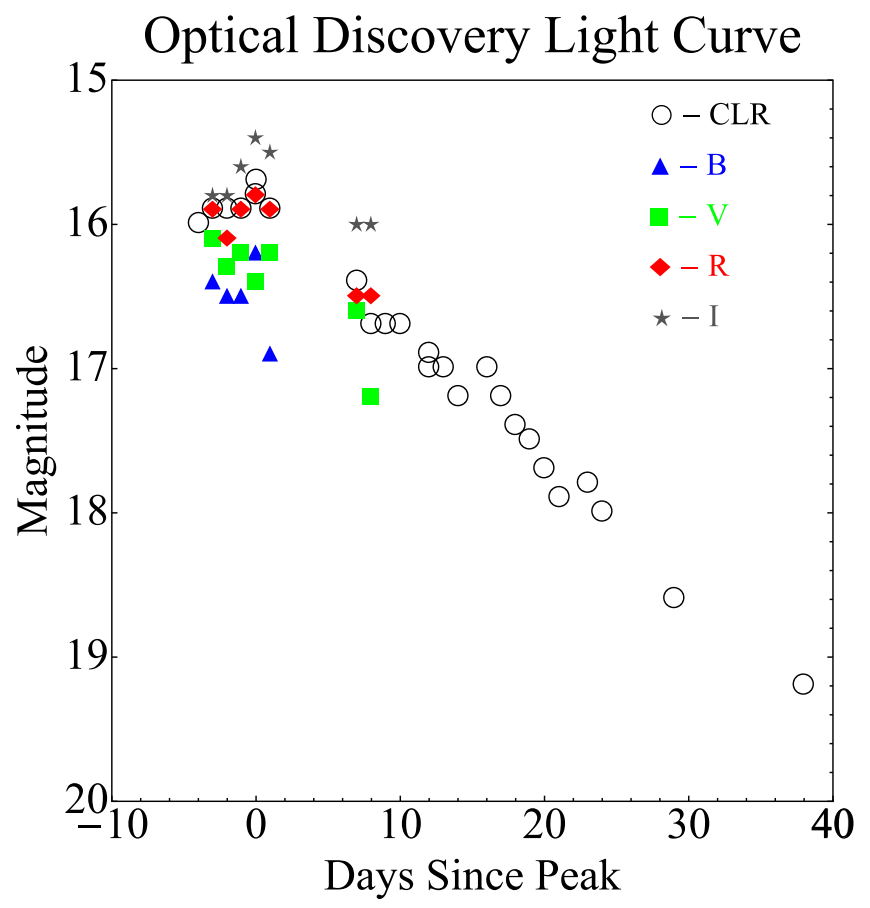

Figure 2. Multiband Optical discovery light curve of SN 2010da taken from Bronberg Observatory. The peak at $t=0$ corresponds to the time of peak optical brightness ( $\mathrm{JD}=2455343.66)$.

aperture photometry on 11 sets of $B V R I$ frames obtained on four photometric nights, calibrated to standard stars of Landolt (1992). This calibration yielded $V=13.710, B-V=0.596$, $V-R=0.346$, and $V-I=0.675$, with mean errors of about $\pm 0.003-0.007$ mag.

The resulting calibrated magnitudes of the OT from the SMARTS monitoring are listed in Table 2 and are shown in Figures 3(B), 4(A), and (B). The tabulated uncertainties are based only on photon statistics; the systematic errors due to the zero-point uncertainty and instrumental effects are likely of the order of $\pm 0.01-0.02 \mathrm{mag}$. In addition, the OT lies in a small cluster (Binder et al. 2016), only marginally resolved in the $1.3 \mathrm{~m}$ images, which contaminates the photometry at the OT's faintest level; we have not attempted to correct for this effect.

\subsubsection{Follow-up with Swope}

The $g$-, $r$-, and $i$-band follow-up images were taken between 2014 May 17 to 2015 November 13 with the 1m-Swope telescope, at Las Campanas Observatory, using a recently installed e2v CCD, $4 \mathrm{~K} \times 4 \mathrm{~K}$ pixels array of 0 !" $435 /$ pix. The observations, the standard data reduction procedure (bias subtraction, flat fielding, and linearity correction) and the photometry calibration was performed by Carnegie Supernova Project collaborators in a similar way as detailed in Hamuy et al. (2006). The field was calibrated in two photometric nights: 2014 August 1 and 2014 December 20. The PSF photometry of SN 2010da were converted from the Natural system of Swope (Contreras et al. 2010) to AB magnitudes based on the transformations provided by Equations (A5)-(A7) in Stritzinger et al. (2011). The following color terms (CT) were adopted in these transformations: $\mathrm{CT}_{g}=-0.010$, $\mathrm{CT}_{r}=-0.014$, and $\mathrm{CT}_{i}=+0.018$. Since the transformed $\mathrm{AB}$ magnitudes of $\mathrm{SN}$ 2010da were consistent within $<0.01 \mathrm{mag}$ of the Natural system photometry, the Natural magnitudes were treated as $\mathrm{AB}$ magnitudes when ultimately converting to the Vega system presented in Table 2. The gri photometry is shown in Figures 3(B) and 4(C).

\subsection{Mid-infrared Observations with Spitzer}

The SPitzer InfraRed Intensive Transient Survey (SPIRITS; M. M. Kasliwal et al. 2016, submitted to ApJ; Tinyanont et al. 2016) targets 194 nearby galaxies within $20 \mathrm{Mpc}$ to a depth of 20 mags on the Vega system. The observations are performed in the $3.6 \mu \mathrm{m}$ and $4.5 \mu \mathrm{m}$ bands of the InfraRed Array Camera (IRAC, Fazio et al. 2004) on board the warm Spitzer Space Telescope (Werner et al. 2004; Gehrz et al. 2007).

The mid-IR counterpart to the SN 2010da, designated SPIRITS 14bme, was identified by SPIRITS in Spitzer/IRAC imaging with channel $1(3.6 \mu \mathrm{m})$ and channel $2(4.5 \mu \mathrm{m})$ taken on 2014 September 5. SPIRITS 14bme is located at an R.A. and decl. of 00:55:04.87 and $-37: 41: 43.8$, within $<0$ "' 2 of the optical counterpart (Monard 2010). The progenitor is detected in archival IRAC taken with channels $1-4$ (3.6, 4.5, 5.8, and $8.0 \mu \mathrm{m}$ ) on 2003 November 21, 2007 December 28.88, and 2007 December 28.94 before the warm mission. All of the available channel 1 and 2 imaging data of SN 2010da/ SPIRITS 14bme from the Spitzer Heritage Archive taken between 2003 November and 2016 March were incorporated in this paper.

To obtain magnitudes, forced aperture photometry was performed on the Post Basic Calibrated Data. The flux was summed in a 4 pixel aperture centered at the SPIRITS-determined coordinates of SPIRITS 14bme. Sky background was measured within an annulus from 8 to 16 pixels surrounding each source and subtracted from the total flux. Finally, fluxes were converted to magnitudes using the Warm Spitzer/IRAC zero points of 18.8024 (channel 1) and 18.3174 (channel 2), along with aperture corrections of 1.21 and 1.22 , respectively, as specified by the IRAC instrument handbook. The channel 1 and 2 fluxes of all available IRAC imaging of SN 2010da/SPIRITS 14bme are provided (in $\mu \mathrm{Jy}$ ) in Table 3, and the photometry is shown in Figure 3(A). The channel 3 and 4 fluxes of the SN 2010da progenitor taken on 2003 November 21 are $87.93 \pm 28.30 \mu \mathrm{Jy}$ and $54.89 \pm 25.00 \mu \mathrm{Jy}$, respectively. The progenitor was also observed with the Multiband-Imaging Photometer for Spitzer (MIPS) on 2003 November 21 from which an upper limit of $\lesssim 136 \mathrm{Jy}$ at $24 \mu \mathrm{m}$ is derived.

In the following sections of this paper, we refer to SPIRITS 14bme as SN 2010da or its mid-IR counterpart.

\subsection{Near-infrared Observations}

The goal of the SPIRITS campaign at the University of Minnesota's Mount Lemmon Observing Facility (MLOF; Low et al. 2007) in Arizona is two-fold. First, transients of interest from the space-based SPIRITS observations are followed up in the $\mathrm{J}(1.25 \mu \mathrm{m}), \mathrm{H}(1.65 \mu \mathrm{m})$ and $\mathrm{K}_{\mathrm{s}}(2.15 \mu \mathrm{m})$ IR bands using a 2MASS imaging camera (Milligan et al. 1996; Skrutskie et al. 2006) mounted on the $60^{\prime \prime}$ telescope at MLOF. This provides additional photometric data on the temporal light curves for these objects. second, a general survey of potential SPIRITS targets are imaged on the 2MASS detector, providing potential information on the progenitors of future SPIRITS objects, and possibly even the discovery of new objects should they be luminous enough for ground-based observations. Sixty minutes of short exposure images of the targeted SPIRITS 
Table 1

SN 2010da Optical Discovery Magnitudes

\begin{tabular}{|c|c|c|c|c|c|c|c|c|c|c|}
\hline JD & $t_{\mathrm{d}}$ & Unfiltered Mag. & $t_{\mathrm{d}}$ & $B$ & $t_{\mathrm{d}}$ & $V$ & $t_{\mathrm{d}}$ & $R$ & $t_{\mathrm{d}}$ & I \\
\hline 2455339.67 & -3.995 & 16. & -3.019 & 16.4 & -3.023 & 16.1 & -3.022 & 15.9 & -3.02 & 15.8 \\
\hline 2455340.64 & -3.025 & 15.9 & -2.016 & 16.5 & -2.019 & 16.3 & -2.018 & 16.1 & -2.017 & 15.8 \\
\hline 2455341.64 & -2.025 & 15.9 & -1.052 & 16.5 & -1.055 & 16.2 & -1.054 & 15.9 & -1.053 & 15.6 \\
\hline 2455342.61 & -1.05 & 15.9 & -0.042 & 16.2 & -0.045 & 16.4 & -0.044 & 15.8 & -0.043 & 15.4 \\
\hline 2455343.61 & -0.05 & 15.8 & 0.96 & 16.9 & 0.956 & 16.2 & 0.957 & 15.9 & 0.958 & 15.5 \\
\hline 2455343.66 & 0. & 15.7 & & & 6.97 & 16.6 & 6.971 & 16.5 & 6.972 & 16.0 \\
\hline 2455344.62 & 0.953 & 15.9 & & & 7.954 & 17.2 & 7.956 & 16.5 & 7.957 & 16.0 \\
\hline 2455350.64 & 6.974 & 16.4 & $\cdots$ & $\cdots$ & $\ldots$ & $\cdots$ & $\cdots$ & $\ldots$ & $\cdots$ & \\
\hline 2455351.62 & 7.959 & 16.7 & $\cdots$ & $\cdots$ & $\cdots$ & $\cdots$ & $\cdots$ & $\cdots$ & $\cdots$ & \\
\hline 2455352.62 & 8.958 & 16.7 & $\cdots$ & $\cdots$ & $\cdots$ & $\cdots$ & $\cdots$ & $\cdots$ & $\cdots$ & \\
\hline 2455353.64 & 9.973 & 16.7 & $\cdots$ & $\cdots$ & $\cdots$ & $\cdots$ & $\cdots$ & $\cdots$ & $\cdots$ & \\
\hline 2455355.63 & 11.966 & 16.9 & $\cdots$ & $\cdots$ & $\cdots$ & $\cdots$ & $\cdots$ & $\cdots$ & $\cdots$ & \\
\hline 2455355.63 & 11.966 & 17. & $\cdots$ & $\cdots$ & $\cdots$ & $\cdots$ & $\cdots$ & $\cdots$ & $\cdots$ & \\
\hline 2455356.64 & 12.976 & 17. & $\cdots$ & $\cdots$ & $\cdots$ & $\cdots$ & $\cdots$ & $\cdots$ & $\cdots$ & \\
\hline 2455357.66 & 13.996 & 17.2 & $\cdots$ & $\cdots$ & $\cdots$ & $\cdots$ & $\cdots$ & $\cdots$ & $\cdots$ & \\
\hline 2455359.67 & 16.002 & 17. & $\cdots$ & $\cdots$ & $\cdots$ & $\cdots$ & $\cdots$ & $\cdots$ & $\cdots$ & \\
\hline 2455360.62 & 16.959 & 17.2 & $\cdots$ & $\cdots$ & $\cdots$ & $\cdots$ & $\cdots$ & $\cdots$ & $\cdots$ & \\
\hline 2455361.62 & 17.953 & 17.4 & $\cdots$ & $\cdots$ & $\cdots$ & $\cdots$ & $\cdots$ & $\cdots$ & $\cdots$ & \\
\hline 2455362.61 & 18.942 & 17.5 & $\cdots$ & $\cdots$ & $\cdots$ & $\cdots$ & $\cdots$ & $\cdots$ & $\cdots$ & \\
\hline 2455363.62 & 19.956 & 17.7 & $\cdots$ & $\cdots$ & $\cdots$ & $\cdots$ & $\cdots$ & $\cdots$ & $\cdots$ & \\
\hline 2455364.67 & 21.004 & 17.9 & $\cdots$ & $\cdots$ & $\cdots$ & $\cdots$ & $\cdots$ & $\cdots$ & $\cdots$ & \\
\hline 2455366.64 & 22.976 & 17.8 & $\cdots$ & $\cdots$ & $\cdots$ & $\cdots$ & $\cdots$ & $\cdots$ & $\cdots$ & \\
\hline 2455367.64 & 23.977 & 18. & $\cdots$ & $\cdots$ & $\cdots$ & $\cdots$ & $\cdots$ & $\cdots$ & $\cdots$ & \\
\hline 2455372.63 & 28.964 & 18.6 & $\cdots$ & $\cdots$ & $\cdots$ & $\cdots$ & $\cdots$ & $\cdots$ & $\cdots$ & \\
\hline 2455381.61 & 37.941 & 19.2 & $\cdots$ & $\cdots$ & $\cdots$ & $\cdots$ & $\cdots$ & $\cdots$ & $\cdots$ & \\
\hline
\end{tabular}

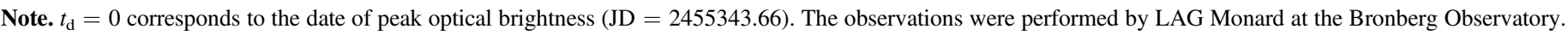

objects are obtained with the 2MASS camera, along with 60 minutes of images of surrounding sky. Using these sky images to remove background radiation, the science images are stacked, co-added, and analyzed to obtain photometric data. Limiting magnitudes with respect to the background are typically 16-17 mag for $\mathrm{H}$ and $\mathrm{K}_{\mathrm{s}}$ bands, and 17-19 mag for the $\mathrm{J}$ band.

$\mathrm{J}, \mathrm{H}$, and $\mathrm{K}_{\mathrm{s}}$ images of NGC 300 were taken at MLOF on 2014 November 23 and 2015 November 22. On 2014 November 23, the $\mathrm{J}, \mathrm{H}$, and $\mathrm{K}_{\mathrm{s}}$ magnitudes for SN 2010da were $17.5 \pm 0.2,16.6 \pm 0.3$, and $16.1 \pm 0.4$, respectively. On 2015 November 22, the $\mathrm{J}, \mathrm{H}$, and $\mathrm{K}_{\mathrm{s}}$ magnitudes were $18.0 \pm 0.3,17.3 \pm 0.4$, and $16.7 \pm 0.5$, respectively.

Near-IR $J$ and $K_{s}$ images of NGC 300 were also obtained on 2014 December 24 with the FourStar infrared camera (Persson et al. 2013) on the Magellan Baade Telescope at Las Campanas Observatory in Chile. The measured $\mathbf{J}$ and $\mathrm{K}_{\mathrm{s}}$ magnitudes for SN 2010da from FourStar were $18.1 \pm 0.3$ and $16.5 \pm 0.2$, respectively.

\section{RESULTS AND ANALYSIS}

\subsection{SN 2010da Light Curves}

\subsubsection{Mid-infrared Photometry}

The full mid-IR light curve of SN 2010da taken by Spitzer/ IRAC before, during, and after the optical and X-ray outburst is shown in Figure 3(A), where $t_{d}=0$ corresponds to the day of the optical peak (JD = 2455343.66). The optical light curve (Figure 3(B)) and X-ray observations (Figure 3(C)) from Binder et al. (2016, and references therein) are plotted on the same time axis as the mid-IR light curve. On day -156 , the $3.6 \mu \mathrm{m}$ emission increased by $\sim 50 \%$ of the progenitor flux as measured from day -2379 and -880 observations. This flux increase is significant given that the source has remained constant to within a few percent at 3.6 and within $\sim 10 \%$ at $4.5 \mu \mathrm{m}$ between days -2379 and -880 . Within 23 days of the initial brightening on day -156 , the $3.6 \mu \mathrm{m}$ flux increased to its highest measured mid-IR value of $0.27 \mathrm{mJy}$, a factor of $\sim 2.5$ times the progenitor flux. At day 60, after the initial optical outburst, the source exhibited a similar $3.6 \mu \mathrm{m}$ flux as on day -133 . The sharp increase and immediate decrease in brightness at $3.6 \mu \mathrm{m}$ indicates that an outburst in the mid-IR occurred between days -133 and 81 , which is consistent with the timing of the X-ray and optical emission peaks.

By day 460 , the 3.6 and $4.5 \mu \mathrm{m}$ flux decreased to values slightly higher and lower than the progenitor flux at respective wavelengths. The following measurement made on day 806 indicated a gradual re-brightening in the mid-IR with similar fluxes observed on day 1386. Between days 1386 and 1602, there is evidence of rapid re-brightening given the sharp increase in flux from day 1386 to 1562 followed by a steep decrease. At day 1936, the mid-IR flux increased by $\sim 25 \%$ from day 1602 and decreased slightly in the subsequent observation on day 1943. This is suggestive of another rebrightening event in the mid-IR between days 1602 and 1936 and, as in the case of the initial outburst, appears to coincide with the re-brightening X-ray and optical emission (blue dashed and green dot-dashed lines in Figure 3, respectively). The following two observations on days 1951 and 1965 show a rapid increase to the peak measured brightness at late-times, almost a factor of approximately two greater than the progenitor flux. The most recent two mid-IR observations made on days 2097 and 2104 show a sharp decrease in flux $\sim 10 \%$ lower than on day 1965 , which suggests that another 
Table 2

Optical Magnitudes, Color, and Luminosity

\begin{tabular}{|c|c|c|c|c|c|c|c|c|c|}
\hline JD & $t_{\mathrm{d}}$ & $B$ & $V / g$ & $R / r$ & $I / i$ & $B-V$ & $V-I / g-i$ & $\log \left(L / L_{\odot}\right)$ & $T(\mathrm{~K})$ \\
\hline 2455341.86 & -1.803 & $16.69(0.01)$ & $16.34(0.01)$ & $15.97(0.01)$ & $15.68(0.01)$ & $0.36(0.01)$ & $0.66(0.01)$ & $6.4(0)$. & $99390(100)$ \\
\hline 2455341.87 & -1.799 & $16.7(0.02)$ & $16.31(0.02)$ & $15.94(0.01)$ & $15.68(0.02)$ & $0.38(0.02)$ & $0.64(0.02)$ & $6.4(0.01)$ & $9360(190)$ \\
\hline 2455342.88 & -0.785 & $16.6(0.02)$ & $16.24(0.01)$ & $15.88(0.03)$ & $15.6(0.04)$ & $0.36(0.02)$ & $0.64(0.04)$ & $6.44(0)$. & $9470(290)$ \\
\hline 2455343.86 & 0.194 & $16.55(0.03)$ & $16.17(0.02)$ & $15.81(0.01)$ & $15.5(0.02)$ & $0.38(0.03)$ & $0.66(0.03)$ & $6.46(0.02)$ & $9230(240)$ \\
\hline 2455346.89 & 3.224 & $16.83(0.01)$ & $16.46(0.01)$ & $16.02(0.01)$ & $15.78(0.01)$ & $0.37(0.01)$ & $0.68(0.01)$ & $6.34(0.01)$ & $9200(110)$ \\
\hline 2455347.9 & 4.231 & $17.08(0.02)$ & $16.67(0.02)$ & $16.17(0.02)$ & $15.98(0.02)$ & $0.41(0.03)$ & $0.69(0.03)$ & $6.24(0.02)$ & $8930(240)$ \\
\hline 2455348.87 & 5.206 & $17.27(0.01)$ & $16.85(0.01)$ & $16.31(0.01)$ & $16.08(0.01)$ & $0.42(0.02)$ & $0.77(0.02)$ & $6.15(0.01)$ & $8460(110)$ \\
\hline 2455350.88 & 7.22 & $17.41(0)$. & $\ldots$ & $16.42(0)$. & $\ldots$ & $\ldots$ & $\ldots$ & $\ldots$ & $\ldots$ \\
\hline 2455350.91 & 7.247 & $17.4(0.02)$ & $16.97(0.01)$ & $16.41(0.01)$ & $16.18(0.02)$ & $0.43(0.02)$ & $0.79(0.02)$ & $6.1(0.01)$ & $8330(120)$ \\
\hline 2455351.86 & 8.199 & $17.56(0.01)$ & $17.12(0.01)$ & $16.52(0.01)$ & $16.28(0.01)$ & $0.44(0.01)$ & $0.84(0.01)$ & $6.04(0.01)$ & $8110(80)$ \\
\hline 2455352.87 & 9.201 & $17.64(0.01)$ & $17.21(0.01)$ & $16.57(0.01)$ & $16.34(0.01)$ & $0.43(0.01)$ & $0.87(0.01)$ & 6. $(0.01)$ & $7980(80)$ \\
\hline 2455352.88 & 9.216 & $17.64(0.01)$ & $17.16(0.01)$ & $16.58(0.01)$ & $16.36(0.01)$ & $0.48(0.01)$ & $0.81(0.02)$ & $6.01(0.01)$ & $8060(90)$ \\
\hline 2455353.9 & 10.233 & $17.8(0.01)$ & $17.31(0.01)$ & $16.69(0.01)$ & $16.44(0.01)$ & $0.48(0.01)$ & $0.87(0.01)$ & $5.94(0.01)$ & $7780(70)$ \\
\hline 2455355.85 & 12.189 & 18. $(0.01)$ & $17.55(0.01)$ & $16.84(0.01)$ & $16.63(0.01)$ & $0.46(0.02)$ & $0.92(0.02)$ & $5.86(0.01)$ & $7690(80)$ \\
\hline 2455355.87 & 12.204 & 18. $(0.01)$ & $17.54(0.01)$ & $16.86(0.01)$ & $16.6(0.02)$ & $0.46(0.02)$ & $0.94(0.02)$ & $5.86(0.01)$ & $7560(90)$ \\
\hline 2455358.93 & 15.266 & $18.39(0.01)$ & $17.9(0.02)$ & $17.14(0.01)$ & $16.94(0.02)$ & $0.49(0.02)$ & $0.96(0.03)$ & $5.71(0.01)$ & $7400(100)$ \\
\hline 2455358.94 & 15.273 & $18.4(0.02)$ & $17.95(0.01)$ & $17.16(0.01)$ & $16.92(0.02)$ & $0.45(0.02)$ & $1.03(0.03)$ & $5.7(0.01)$ & $7250(120)$ \\
\hline 2455360.91 & 17.25 & $18.52(0.02)$ & $18.04(0.01)$ & $17.23(0.01)$ & $17.02(0.02)$ & $0.48(0.02)$ & $1.02(0.02)$ & $5.65(0.01)$ & $7220(80)$ \\
\hline 2455364.93 & 21.264 & $18.89(0.02)$ & $18.46(0.02)$ & $17.53(0.02)$ & $17.38(0.02)$ & $0.43(0.03)$ & $1.07(0.03)$ & $5.5(0.02)$ & $7150(110)$ \\
\hline 2455369.85 & 26.181 & $19.18(0.03)$ & $18.72(0.03)$ & $17.8(0.02)$ & $17.61(0.02)$ & $0.46(0.04)$ & $1.12(0.04)$ & $5.39(0.02)$ & $6930(130)$ \\
\hline 2455369.86 & 26.196 & $19.18(0.03)$ & $18.68(0.03)$ & $17.78(0.02)$ & $17.56(0.03)$ & $0.49(0.04)$ & $1.13(0.05)$ & $5.4(0.02)$ & $6810(140)$ \\
\hline 2455372.86 & 29.193 & $19.53(0.06)$ & $19.02(0.06)$ & 18. $(0.03)$ & $17.89(0.04)$ & $0.52(0.09)$ & $1.12(0.07)$ & $5.26(0.05)$ & $6770(200)$ \\
\hline 2455372.87 & 29.201 & $19.45(0.07)$ & $18.96(0.04)$ & $18.01(0.02)$ & $17.85(0.03)$ & $0.49(0.08)$ & $1.12(0.05)$ & $5.29(0.04)$ & $6850(210)$ \\
\hline 2455374.89 & 31.223 & $\ldots$ & $\ldots$ & $17.84(0.03)$ & $17.72(0.04)$ & $\ldots$ & $\ldots$ & $\ldots$ & $\ldots$ \\
\hline 2455374.9 & 31.238 & $\ldots$ & $\ldots$ & $18.18(0.05)$ & 18. $(0.07)$ & $\ldots$ & $\ldots$ & $\ldots$ & $\ldots$ \\
\hline 2455376.87 & 33.201 & $19.52(0.06)$ & $19.11(0.04)$ & $18.1(0.02)$ & $17.93(0.03)$ & $0.41(0.07)$ & $1.17(0.06)$ & $5.25(0.04)$ & $6830(190)$ \\
\hline 2455377.95 & 34.29 & $19.62(0.06)$ & $19.24(0.11)$ & $18.29(0.06)$ & $17.96(0.06)$ & $0.38(0.12)$ & $1.27(0.12)$ & $5.21(0.06)$ & $6570(180)$ \\
\hline 2455378.82 & 35.157 & $19.67(0.06)$ & $19.18(0.04)$ & $18.24(0.02)$ & $18.01(0.03)$ & $0.5(0.07)$ & $1.16(0.05)$ & $5.2(0.04)$ & $6690(170)$ \\
\hline 2455380.84 & 37.178 & $19.78(0.04)$ & $19.26(0.03)$ & $18.29(0.02)$ & $18.1(0.03)$ & $0.52(0.05)$ & $1.15(0.04)$ & $5.17(0.03)$ & $6670(140)$ \\
\hline 2455380.86 & 37.193 & $19.7(0.04)$ & $19.3(0.04)$ & $18.28(0.03)$ & $18.15(0.04)$ & $0.4(0.06)$ & $1.15(0.06)$ & $5.17(0.04)$ & $6960(210)$ \\
\hline 2455382.79 & 39.121 & $19.8(0.04)$ & $19.35(0.03)$ & $18.36(0.02)$ & $\ldots$ & $0.45(0.06)$ & $\ldots$ & $\ldots$ & $\ldots$ \\
\hline 2455385.9 & 42.239 & 20. $(0.03)$ & $19.49(0.02)$ & $18.52(0.02)$ & $\ldots$ & $0.51(0.04)$ & $\ldots$ & $\ldots$ & $\ldots$ \\
\hline 2455385.92 & 42.252 & $20.05(0.04)$ & $19.56(0.05)$ & $18.53(0.03)$ & $18.34(0.04)$ & $0.49(0.06)$ & $1.22(0.06)$ & $5.06(0.03)$ & $6530(140)$ \\
\hline 2455388.92 & 45.26 & $20.15(0.03)$ & $19.63(0.03)$ & $18.64(0.02)$ & & $0.51(0.04)$ & $\ldots$ & $\ldots$ & $\ldots$ \\
\hline 2455390.86 & 47.197 & $20.22(0.02)$ & $19.74(0.02)$ & $18.72(0.02)$ & $18.51(0.02)$ & $0.48(0.03)$ & $1.24(0.03)$ & $4.99(0.02)$ & $6490(90)$ \\
\hline 2455410.84 & 67.176 & $\ldots$ & $\ldots$ & $19.17(0.04)$ & $18.88(0.04)$ & $\ldots$ & $\ldots$ & $\ldots$ & $\ldots$ \\
\hline 2455417.87 & 74.205 & $\ldots$ & $\ldots$ & $19.39(0.04)$ & $19.06(0.04)$ & $\ldots$ & $\ldots$ & $\ldots$ & $\ldots$ \\
\hline 2455421.79 & 78.126 & $20.83(0.04)$ & $20.38(0.04)$ & $19.33(0.04)$ & $19.06(0.04)$ & $0.45(0.06)$ & $1.32(0.06)$ & $4.74(0.03)$ & $6270(130)$ \\
\hline 2455425.78 & 82.118 & $21.07(0.05)$ & $20.64(0.04)$ & $19.52(0.04)$ & $19.18(0.04)$ & $0.43(0.06)$ & $1.45(0.06)$ & $4.66(0.03)$ & 5940 (130) \\
\hline 2455430.81 & 87.15 & $\ldots$ & $\ldots$ & $19.52(0.05)$ & $19.37(0.06)$ & $\ldots$ & $\ldots$ & $\ldots$ & $\ldots$ \\
\hline 2455444.82 & 101.16 & $21.3(0.06)$ & $20.83(0.05)$ & $19.79(0.05)$ & $19.49(0.06)$ & $0.47(0.07)$ & $1.34(0.07)$ & $4.56(0.04)$ & 6180 (190) \\
\hline 2455877.62 & 533.956 & $19.84(0.06)$ & $19.27(0.04)$ & $18.5(0.04)$ & $18.15(0.03)$ & $0.58(0.07)$ & $1.12(0.05)$ & $5.15(0.04)$ & $6660(150)$ \\
\hline 2455885.63 & 541.965 & $20.35(0.03)$ & $19.81(0.03)$ & $18.89(0.03)$ & $18.49(0.03)$ & $0.54(0.05)$ & $1.32(0.04)$ & $4.96(0.02)$ & $6120(100)$ \\
\hline 2455893.59 & 549.927 & $20.78(0.05)$ & $20.22(0.04)$ & $19.33(0.04)$ & $18.9(0.04)$ & $0.56(0.06)$ & $1.32(0.06)$ & $4.79(0.03)$ & 6080 (130) \\
\hline 2455895.55 & 551.89 & $21.28(0.07)$ & $20.39(0.05)$ & $\ldots$ & $\ldots$ & $0.89(0.09)$ & $\ldots$ & $\ldots$ & $\ldots$ \\
\hline 2455896.61 & 552.944 & $21.34(0.08)$ & $20.38(0.06)$ & $\ldots$ & $\ldots$ & $0.96(0.1)$ & $\ldots$ & $\ldots$ & $\ldots$ \\
\hline 2455900.62 & 556.956 & $\ldots$ & $\ldots$ & $19.83(0.09)$ & $19.13(0.07)$ & $\ldots$ & $\ldots$ & $\ldots$ & $\ldots$ \\
\hline 2455909.6 & 565.939 & $\ldots$ & $\ldots$ & $20.09(0.1)$ & $19.4(0.08)$ & $\ldots$ & $\ldots$ & $\ldots$ & $\ldots$ \\
\hline 2455916.6 & 572.939 & $\ldots$ & $\ldots$ & $20.18(0.09)$ & $19.43(0.08)$ & $\ldots$ & $\ldots$ & $\ldots$ & $\ldots$ \\
\hline 2456794.94 & 1451.273 & $\ldots$ & $\ldots$ & $19.99(0.17)$ & $20.11(0.21)$ & $\ldots$ & $\ldots$ & $\ldots$ & $\ldots$ \\
\hline 2456828.94 & 1485.279 & $\ldots$ & $21.08(0.17)$ & $19.73(0.08)$ & $\ldots$ & $\ldots$ & $\ldots$ & $\ldots$ & $\ldots$ \\
\hline 2456828.94 & 1485.28 & $\ldots$ & $20.97(0.17)$ & $19.72(0.08)$ & $19.76(0.13)$ & $\ldots$ & $1.22(0.21)$ & $4.74(0.18)$ & 6640 (1100) \\
\hline 2456829.89 & 1486.23 & $\ldots$ & $20.89(0.14)$ & $19.68(0.07)$ & $19.74(0.14)$ & $\ldots$ & $1.15(0.2)$ & $4.78(0.19)$ & 6920 (1130) \\
\hline 2456870.79 & 1527.129 & $\ldots$ & $20.42(0.08)$ & $19.47(0.06)$ & $19.51(0.09)$ & $\ldots$ & $0.91(0.12)$ & $4.96(0.22)$ & $8150(980)$ \\
\hline 2456870.79 & 1527.13 & $\ldots$ & $20.53(0.09)$ & $19.46(0.06)$ & $19.44(0.08)$ & $\ldots$ & $1.09(0.12)$ & $4.92(0.2)$ & $7170(760)$ \\
\hline 2456871.85 & 1528.186 & $\ldots$ & $20.67(0.1)$ & $19.54(0.06)$ & $19.72(0.1)$ & $\ldots$ & $0.96(0.14)$ & $4.86(0.22)$ & 7860 (1080) \\
\hline 2456871.85 & 1528.187 & $\ldots$ & $20.83(0.11)$ & $19.59(0.06)$ & $19.85(0.11)$ & $\ldots$ & $0.98(0.15)$ & $4.8(0.22)$ & 7730 (1110) \\
\hline 2456980.56 & 1636.895 & $\ldots$ & $20.74(0.09)$ & $\ldots$ & $\ldots$ & $\ldots$ & $\ldots$ & $\ldots$ & $\ldots$ \\
\hline 2456980.56 & 1636.896 & $\ldots$ & $20.46(0.1)$ & $19.51(0.06)$ & $19.46(0.08)$ & $\ldots$ & 1. $(0.13)$ & $4.94(0.22)$ & $7620(960)$ \\
\hline 2457011.58 & 1667.913 & $\ldots$ & $20.74(0.1)$ & $19.58(0.06)$ & $19.51(0.09)$ & $\ldots$ & $1.23(0.13)$ & $4.83(0.17)$ & $6570(710)$ \\
\hline 2457011.58 & 1667.915 & $\ldots$ & $20.72(0.1)$ & $19.49(0.06)$ & $19.58(0.09)$ & $\ldots$ & $1.14(0.13)$ & $4.84(0.19)$ & $6950(790)$ \\
\hline 2457176.91 & 1833.243 & $\ldots$ & $20.71(0.23)$ & $19.05(0.08)$ & $19.22(0.1)$ & $\ldots$ & $1.49(0.25)$ & $4.85(0.09)$ & $5730(930)$ \\
\hline 2457176.91 & 1833.244 & $\ldots$ & $20.39(0.15)$ & $19.24(0.08)$ & $19.36(0.11)$ & $\ldots$ & $1.03(0.18)$ & $4.98(0.22)$ & 7490 (1220) \\
\hline 2457222.82 & 1879.153 & $\ldots$ & $20.43(0.08)$ & $19.34(0.05)$ & $19.32(0.07)$ & $\ldots$ & $1.1(0.11)$ & $4.96(0.2)$ & $7120(710)$ \\
\hline
\end{tabular}


Table 2

(Continued)

\begin{tabular}{|c|c|c|c|c|c|c|c|c|c|}
\hline JD & $t_{\mathrm{d}}$ & $B$ & $V / g$ & $R / r$ & $I / i$ & $B-V$ & $V-I / g-i$ & $\log \left(L / L_{\odot}\right)$ & $T(\mathrm{~K})$ \\
\hline 2457222.82 & 1879.155 & $\ldots$ & $20.37(0.08)$ & $19.27(0.05)$ & $19.27(0.08)$ & $\cdots$ & $1.1(0.11)$ & $4.98(0.2)$ & $7130(680)$ \\
\hline 2457247.88 & 1904.217 & $\cdots$ & $20.44(0.08)$ & $19.22(0.05)$ & $19.35(0.08)$ & $\cdots$ & $1.09(0.12)$ & $4.95(0.2)$ & $7170(740)$ \\
\hline 2457247.88 & 1904.219 & $\cdots$ & $20.36(0.07)$ & $19.32(0.05)$ & $19.23(0.07)$ & $\ldots$ & $1.13(0.09)$ & $4.99(0.19)$ & $7020(590)$ \\
\hline 2457339.67 & 1996.008 & $\ldots$ & $20.49(0.09)$ & $19.38(0.05)$ & $19.5(0.09)$ & $\ldots$ & $0.99(0.13)$ & $4.94(0.22)$ & $7690(930)$ \\
\hline
\end{tabular}

Note. Numbers in the parentheses correspond to the $1 \sigma$ uncertainty of the adjacent value. Entries with " 0 " indicate errors less than $<0.01$. Empty entries (“ $\ldots$ ") designate observations where SN 2010da was not detected, or the data are bad/non-existent. $t_{\mathrm{d}}=0$ corresponds to the date of peak optical brightness from the optical discovery light curve (JD $=2455343.66)$.
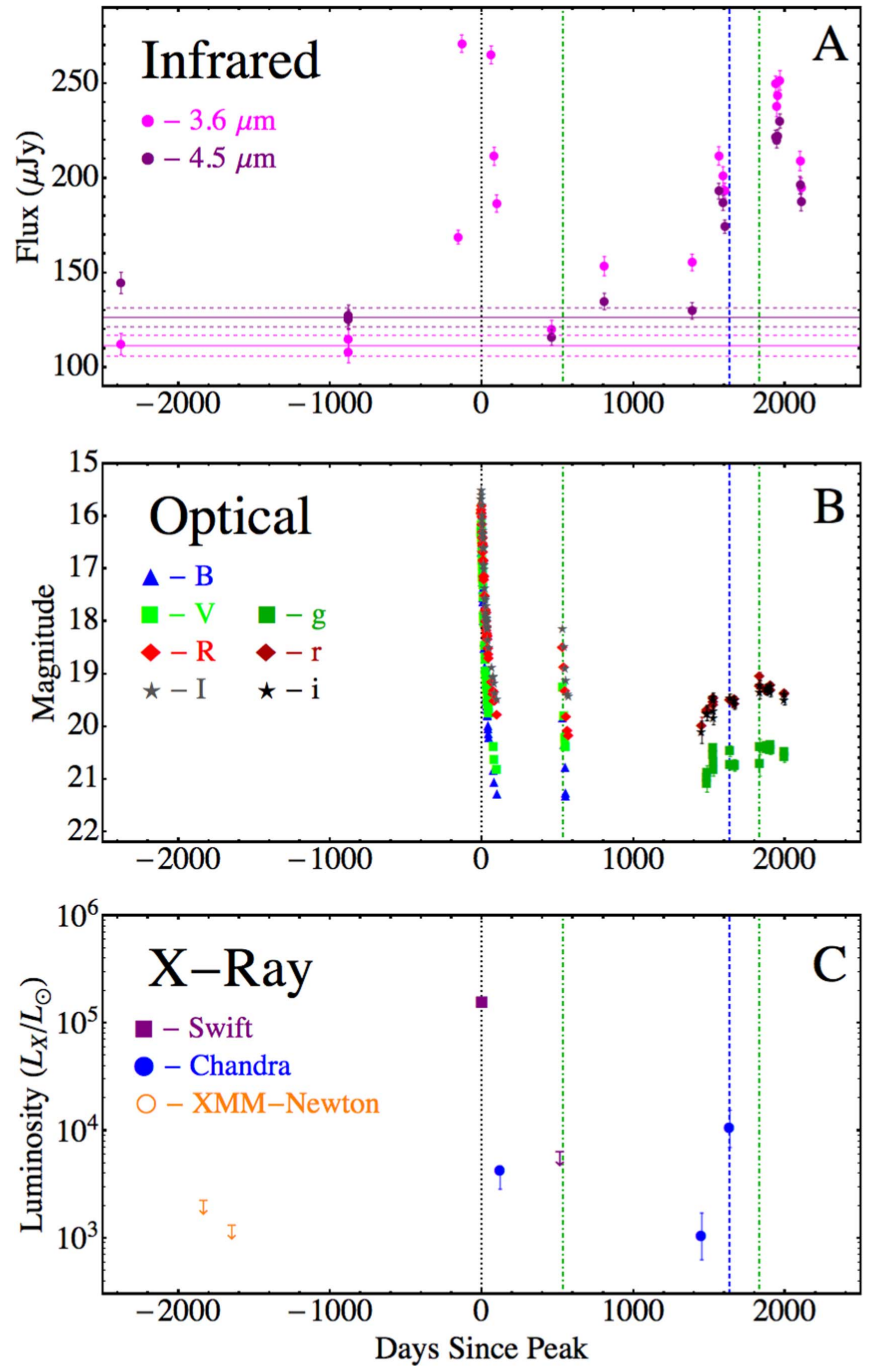

Figure 3. Mid-IR (A), optical (B), and X-ray (C) light curve of SN 2010da. X-ray data are from Binder et al. (2016, and references therein). Green dotteddashed lines and blue dashed lines correspond to observed re-brightening peaks in optical and X-ray emission, respectively.

peak in the mid-IR emission occurred between days 1965 and 2097.

\subsubsection{Optical Photometry}

Detailed BVRI light curves of SN 2010da taken at "early" (day $\sim 0-100$ ) and "intermediate" (day 500) epochs and the gri light curve taken at a "late" (day 1300-2000) epoch are shown in Figures 4(A)-(C), respectively. Prior to its discovery as an OT (Monard 2010), archival optical imaging of NGC 300 from Magellan/Megacam reveal non-detections of $\mathrm{r}$ - and i-band counterparts to a deep limit of $24 \mathrm{AB}$ mag (Berger \& Chornock 2010). The early epoch observations capture a peak in the optical emission occurring 4.2 days after its discovery followed by a rapid decline in brightness by a factor of $\sim 20$ from the peak flux within 50 days, and a factor of $\sim 60$ within 100 days. Given the peak V-band flux of $16.17 \pm 0.02 \mathrm{mag}$ and a distance modulus of $\mu=26.5$ (Dalcanton et al. 2009), the absolute visual magnitude of the optical peak is $M_{V}=-10.3$, consistent with the value reported from the initial discovery (Khan et al. 2010; Monard 2010).

The intermediate epoch BVRI light curve (Figure 4(B)) reveals a re-brightening of the optical counterpart to $V=19.27 \pm 0.04 \mathrm{mag}$, which is $\sim 3.0 \mathrm{mag}$ fainter than the peak brightness but $\sim 1.5 \mathrm{mag}$ brighter than the final $V$ magnitude measured in the early epoch. The timing and brightness of this re-brightening is consistent with the $6.5 \mathrm{~m}$ Magellan/Clay telescope optical observations of SN 2010da reported by Chornock et al. (2011). As in the case of the initial outburst, the intermediate outburst exhibits a steep decline in brightness in the following $\sim 50$ days.

The late-time gri light curve (Figure 4(C)) exhibits a gradual re-brightening between days 1530 and 1833 by $\sim 0.5$ mag in all three bands. Only the $r$ band, however, exceeds the lowest measured brightness in the earlier epochs assuming $r \approx R$, $g \approx V$, and $i \approx I$. The late epoch optical re-brightening is consistent with the timing of the re-brightening X-ray emission observed on day 1635 . In the $\sim 70$ days following the apparent optical peak at day 1833, the optical brightness does not vary significantly. However, the final gri measurements made on day 1996, which is $\sim 30$ days after the final mid-IR observation, show a $\sim 0.2$ mag decrease from the peak brightness.

The evolution of the optical colors $(B-V, V-I$, and $g-i)$ from the outbursts at early, intermediate, and late epochs is shown in Figures 4(D), (E), and (F), respectively. In the early epoch, the $V-I$ color becomes significantly redder within $\sim 20$ days of the initial outburst, increasing from $V-I \approx 0.6$ to 1.0. The $B-V$ color does not exhibit the same evolution and only increases from $B-V \approx 0.38$ at the outburst to 0.45 after 20 days. The optical colors measured at the peak of the intermediate epoch are consistent with the colors in the early epoch after day 40; however, there are less than 10 observations between days 40 and 530. At the final $B$ - and $V$-band measurements of the decreasing brightness after the outburst, the $B-V$ color shows a rapid red-ward change from $B-V \approx 0.48-0.92$ within a short 10 -day timescale. Late-time 

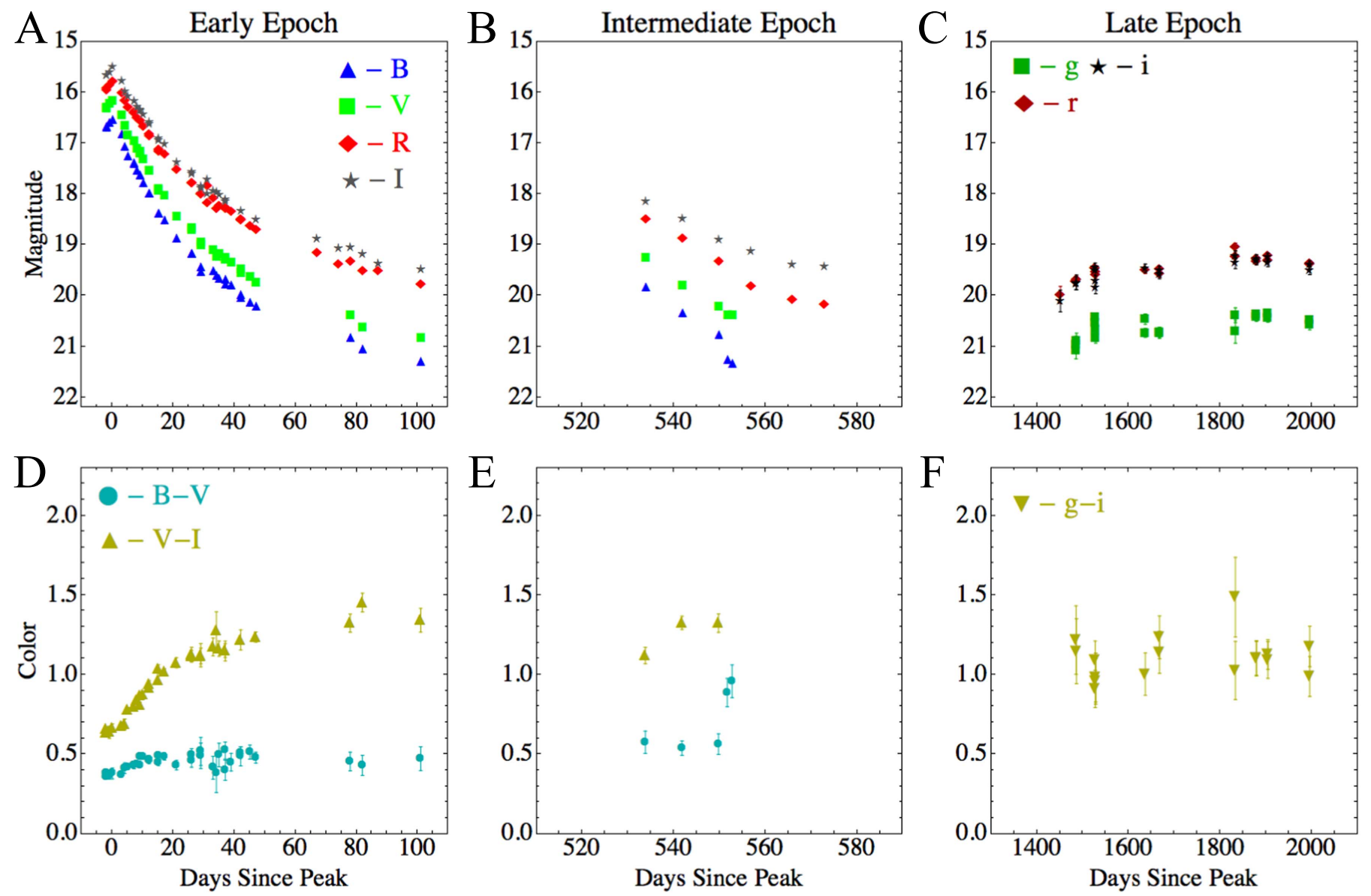

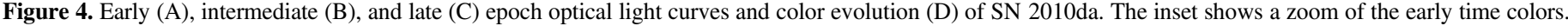

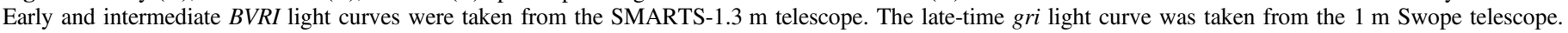

$g-i$ colors are consistent with the $V-I$ colors at intermediate times $(g-i \sim 1.1)$.

\subsection{Properties of the SN 2010da Mid-IR and Optical Counterpart}

\subsubsection{Dust Temperature, IR Luminosity, Mass, and Equilibrium Temperature Radius}

The detection of the progenitor in the mid-IR and nondetection at optical wavelengths are consistent with a central source that was enshrouded by dense and dusty circumstellar material. This mid-IR emission likely originates from hot $\left(T_{d} \gtrsim 500 \mathrm{~K}\right)$ circumstellar material in the vicinity of $\mathrm{SN}$ 2010da and its progenitor and can therefore be used to trace the properties of the emitting dust. The temporal evolution of the dust temperature $\left(T_{d}\right)$, total IR luminosity $\left(L_{\mathrm{IR}}\right)$, and mass $\left(M_{d}\right)$ and the equilibrium temperature radius $\left(R_{\mathrm{Eq}}\right)$ are shown in Figures 5(A)-(D), respectively, and provided in Table 3.

Dust temperatures were derived by fitting the 3.6 and $4.5 \mu \mathrm{m}$ fluxes to a single-temperature modified blackbody, a Planck function multiplied by a grain emissivity model assuming a composition of carbonaceous $a=0.1 \mu \mathrm{m}$ sized grains (Zubko et al. 2004). The progenitor dust exhibited temperatures of $650-750 \mathrm{~K}$. At the first detection made with at both 3.6 and $4.5 \mu \mathrm{m}$ after the outburst (day 460), the dust temperature increased to $850 \pm 70 \mathrm{~K}$ and showed an increasing trend up until it peaked at day $1386-1020 \pm 90 \mathrm{~K}$. The following measurement taken 175 days after the temperature peak revealed that the temperature quickly decreased to $910 \pm 50 \mathrm{~K}$. No significant deviations from $\sim 900 \mathrm{~K}$ were measured.

The total infrared luminosity of the emitting dust was estimated by integrating over the temperature-fitted modified blackbody and assuming a distance of $2.0 \mathrm{Mpc}$ toward NGC 300 (Dalcanton et al. 2009). The evolution of $L_{\mathrm{IR}}$ closely resembles the mid-IR light curve with a gradual post-outburst increase followed by a decrease measured at day 2097. Prior to the eruption, the progenitor exhibited an IR luminosity of $L_{\mathrm{IR}} \approx 1.4 \pm 0.1 \times 10^{4} L_{\odot}$, consistent with the mid-IR progenitor luminosity determined by Prieto et al. (2010). On day 1936, where measured IR luminosity peaked, $L_{\mathrm{IR}}$ increased by over a factor of two from the progenitor levels to $3.5 \pm 0.3 \times 10^{4} L_{\odot}$. The final measurements made on days 2097 and 2104 show a decrease from the peak luminosity to $\sim 2.5 \pm 0.3 \times 10^{4} L_{\odot}$.

Dust masses were derived from the mid-IR fluxes and temperature-fitted modified blackbodies as indicated in Equation (1):

$$
M_{d}=\frac{(4 / 3) a \rho_{b} F_{\lambda} d^{2}}{Q_{C}(\lambda, a) B_{\lambda}\left(T_{d}\right)},
$$

where $a$ is the adopted grain size, $\rho_{b}$ is the bulk density of the dust grains, $F_{\lambda}$ is the measured flux, $d$ is the distance to the source, $Q_{C}(\lambda, a)$ is the grain emissivity model for carbonaceous grains, and $B_{\lambda}\left(T_{d}\right)$ is the Planck function. A bulk density of 
Table 3

Mid-IR Flux and Dust Properties

\begin{tabular}{|c|c|c|c|c|c|c|c|}
\hline JD & $t_{\mathrm{d}}$ & $F_{3.6}(\mu \mathrm{Jy})$ & $F_{4.5}(\mu \mathrm{Jy})$ & $T_{\mathrm{d}}(\mathrm{K})$ & $L_{\mathrm{IR}}\left(\times 10^{4} L_{\odot}\right)$ & $M_{d}\left(\times 10^{-7} M_{\odot}\right)$ & $\mathrm{R}_{\mathrm{eq}}(\mathrm{AU})$ \\
\hline 2452964.56 & -2379.1 & $112.31(5.63)$ & $144.65(5.68)$ & $646(48)$ & $1.4(0.1)$ & $3.84(1.4)$ & $12.2(2)$. \\
\hline 2454463.38 & -880.286 & $115.05(5.54)$ & $125.17(5.15)$ & $751(66)$ & $1.4(0.2)$ & $1.65(0.6)$ & $8.9(1.7)$ \\
\hline 2454463.44 & -880.223 & $107.91(5.45)$ & $127.65(5.25)$ & $695(57)$ & $1.3(0.1)$ & $2.38(0.9)$ & $10.1(1.8)$ \\
\hline 2455187.38 & -156.287 & $168.96(3.62)$ & $\ldots$ & $\ldots$ & $\ldots$ & $\ldots$ & $\ldots$ \\
\hline 2455210.19 & -133.476 & $271.02(4.6)$ & $\ldots$ & $\ldots$ & $\ldots$ & $\ldots$ & $\ldots$ \\
\hline 2455404.68 & 61.0151 & $265.08(4.72)$ & $\ldots$ & $\ldots$ & $\ldots$ & $\ldots$ & $\ldots$ \\
\hline 2455424.6 & 80.9322 & $211.54(4.74)$ & $\ldots$ & $\ldots$ & $\ldots$ & $\ldots$ & $\ldots$ \\
\hline 2455440.26 & 96.5943 & $186.65(4.53)$ & $\ldots$ & $\ldots$ & $\ldots$ & $\ldots$ & $\ldots$ \\
\hline 2455803.21 & 459.542 & $120.14(4.77)$ & $115.83(4.15)$ & $852(73)$ & $1.5(0.3)$ & $0.91(0.3)$ & $7.3(1.4)$ \\
\hline 2456150.05 & 806.389 & $153.59(5.04)$ & $134.81(4.33)$ & $953(80)$ & $2.2(0.5)$ & $0.71(0.2)$ & $6.9(1.3)$ \\
\hline 2456730.01 & 1386.34 & $155.56(4.36)$ & $129.88(4.35)$ & $1019(88)$ & $2.5(0.6)$ & $0.54(0.1)$ & $6.4(1.2)$ \\
\hline 2456905.74 & 1562.08 & $211.75(4.86)$ & $193.13(4.16)$ & 909 (49) & $2.9(0.3)$ & $1.2(0.2)$ & 8.7 (1.) \\
\hline 2456933.92 & 1590.25 & 200.98 (5.09) & $187.02(3.98)$ & 887 (49) & $2.7(0.3)$ & $1.27(0.2)$ & $8.8(1)$. \\
\hline 2456945.26 & 1601.6 & $193.37(4.01)$ & $174.45(3.47)$ & $921(46)$ & $2.7(0.3)$ & $1.03(0.2)$ & $8.2(0.9)$ \\
\hline 2457279.93 & 1936.26 & $249.64(4.24)$ & $221.77(3.58)$ & 939 (39) & $3.5(0.3)$ & $1.22(0.2)$ & 9. (0.8) \\
\hline 2457287.84 & 1944.17 & $237.62(5.13)$ & $220.15(4.15)$ & $892(43)$ & $3.2(0.3)$ & $1.46(0.2)$ & $9.5(1)$. \\
\hline 2457295.22 & 1951.55 & $243.65(5.29)$ & $221.92(3.73)$ & $910(43)$ & $3.3(0.3)$ & $1.37(0.2)$ & $9.3(0.9)$ \\
\hline 2457309.06 & 1965.4 & $251.61(5.18)$ & $230.2(3.7)$ & $905(40)$ & $3.4(0.3)$ & $1.45(0.2)$ & $9.6(0.9)$ \\
\hline 2457440.6 & 2096.94 & $209.07(5.11)$ & $196.29(4.63)$ & 878 (49) & $2.8(0.3)$ & $1.38(0.3)$ & $9.1(1.1)$ \\
\hline 2457448.08 & 2104.42 & $194.91(5.18)$ & $187.36(4.6)$ & 855 (49) & $2.5(0.3)$ & $1.46(0.3)$ & $9.2(1.1)$ \\
\hline
\end{tabular}

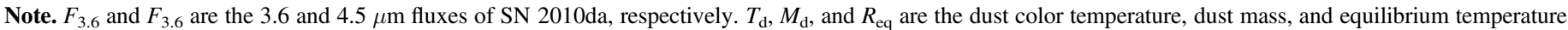

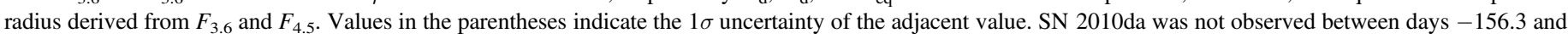
96.6 at $4.5 \mu \mathrm{m}$.

$\rho_{b}=2.2 \mathrm{gm} \mathrm{cm}^{-3}$ (e.g., Draine \& Li 2007) is assumed for the emitting carbonaceous grains. The estimated dust mass of the progenitor averaged over the first three observations $\left(M_{d}=2.6 \pm 1.0 \times 10^{-7} M_{\odot}\right)$ decreased by over a factor of approximately two at the first 3.6 and $4.5 \mu \mathrm{m}$ measurements made after the outburst at day 460 , where $M_{d}=0.9 \pm 0.3 \times 10^{-7} M_{\odot}$. In the following $\sim 930$ days, the dust mass gradually decreased to a minimum of $0.5 \pm 0.1 \times 10^{-7} M_{\odot}$. Between days 1386 and 1562, the dust mass increased significantly by $0.7 \times 10^{-7} M_{\odot}$, which implies that dust was formed at a rate of $\dot{M}_{D} \approx 1.4 \times$ $10^{-7} M_{\odot} \mathrm{yr}^{-1}$. The timing of this increased dust production is consistent with the late-time re-brightening in the X-ray, optical, and mid-IR as well as the decrease in dust temperature. The final mid-IR measurements taken around day 2000 reveal a 20\%-40\% $(1-2 \sigma)$ increase in dust mass from day 1602 .

The equilibrium temperature radius, $R_{\mathrm{Eq}}$, is determined from the distance where the absorbed power from the radiation field of the heating source is equal to the power emitted by the dust grain of temperature $T_{d}$. The equilibrium temperature radius provides an estimate of the physical size of the hot, circumstellar dust and is given by

$$
R_{\mathrm{Eq}} \approx\left(\frac{Q_{\mathrm{abs}}}{Q_{\mathrm{e}}} \frac{L_{\mathrm{IR}}}{16 \pi \sigma T_{D}^{4}}\right)^{1 / 2},
$$

where $Q_{\mathrm{abs}}$ and $Q_{\mathrm{e}}$ are the grain absorption and emission Planck mean cross sections (see Gilman 1974), respectively, and $\sigma$ is the Stefan-Boltzmann constant. A typical value of $\left(Q_{\text {abs }} / Q_{\mathrm{e}}\right) \approx 0.3$ (e.g., Smith et al. 2016) is assumed for the calculation. Note that if the heating of the mid-IR emitting dust is instead dominated locally by collisions with hot electrons in a post-shock environment (e.g., Dwek 1987; Dwek et al. 2008), $R_{\mathrm{Eq}}$ will not reflect the physical size of the system. However, it is unlikely that the mid-IR emitting dust is dominantly heated by a shock driven from the initial outburst since the observed mass of an illuminated shell of shock-heated dust should increase rapidly as a function of time as it propagates outward radially $\left(M_{\mathrm{d}} \propto t^{2}\right.$; Fox et al. 2011). This is inconsistent with the "step-function"-like evolution of the dust mass.

Before the outburst, the progenitor exhibited an equilibrium temperature radius of $\sim 10-12 \mathrm{au}$. After the outburst on day 460 , the radius decreased from the progenitor size to $7.3 \pm 1.4 \mathrm{au}$ and did not change significantly in the following measurements made on days 806 and 1386. At late times coincident with the multi-wavelength outbursts and dust mass increase, the radius grew to $9 \pm 1$ au and did not deviate significantly throughout the final observations.

\subsubsection{Optical Counterpart Temperature and Luminosity}

The evolution of the temperature and luminosity of the dereddened SN 2010da optical counterpart is shown in Figures 6(A) and (B), respectively, and are provided in Table 2. Optical photometry was corrected for foreground extinction $\left(A_{V}=0.26\right)$ and the differential extinction internal to NGC 300 along the line of sight toward SN 2010da $\left(A_{V} \sim 0.4\right.$; Binder et al. 2016).

Temperatures were derived by fitting a single-temperature blackbody to the $B V I$ and $g i$ photometry, where the $R$ and $r$ bands are purposely ignored due to potential contamination from prominent $H \alpha$ line emission detected from the outburst (Chornock \& Berger 2010). Temperatures peak at $\sim 9500 \mathrm{~K}$ for several days around the initial optical peak and decrease rapidly to $\sim 6500 \mathrm{~K}$ in the 40 days following the peak. At the intermediate epoch, the temperature peaks at $\sim 6700 \mathrm{~K}$ on day 534 and decreases to $\sim 6200 \mathrm{~K}$ within eight days. Temperatures derived in the late epoch have large error bars due to the faintness of the optical counterpart; however, they exhibit a range of temperature $\sim 6000-8000 \mathrm{~K}$ consistent with values estimated during the previous epochs. 
A

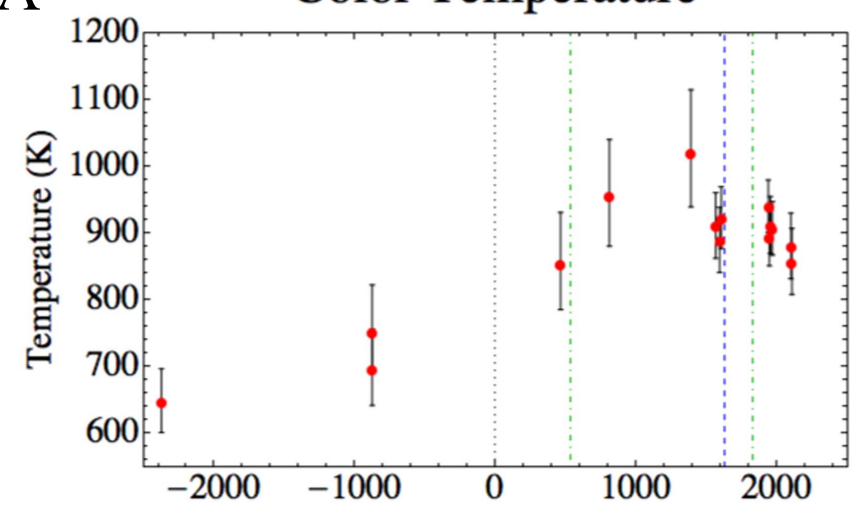

C

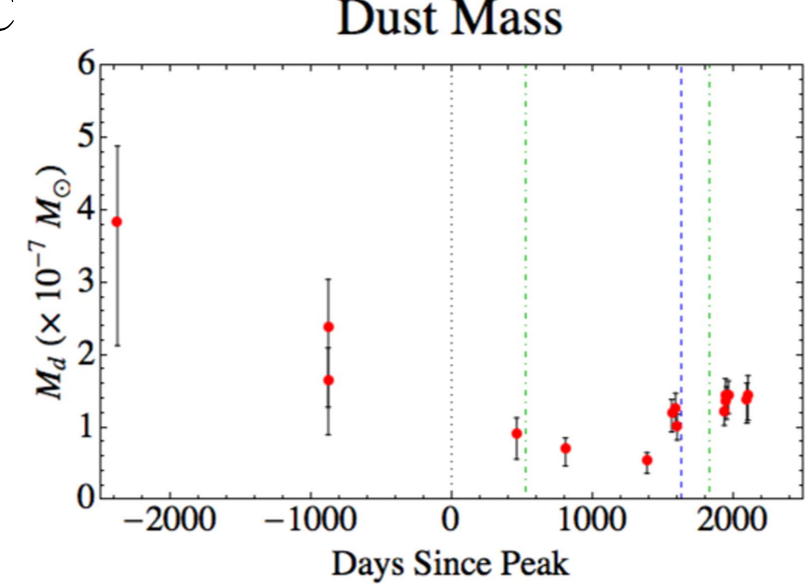

B

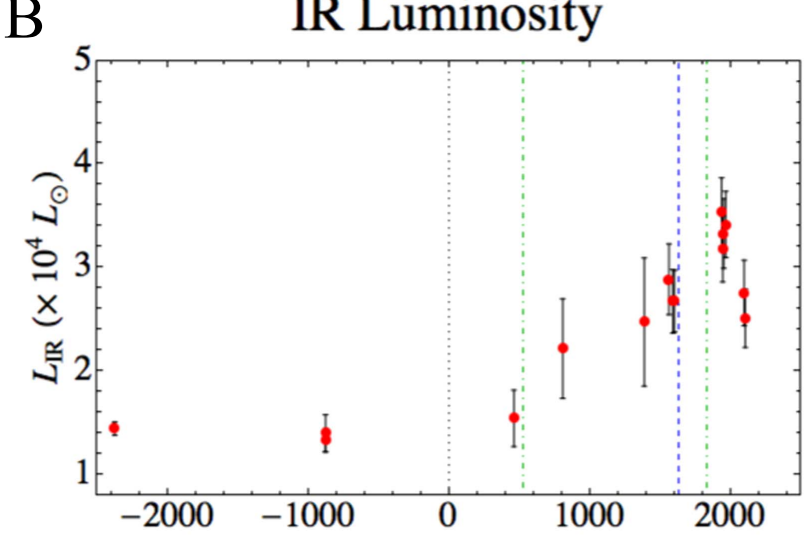

D Equilibrium Temperature Radius

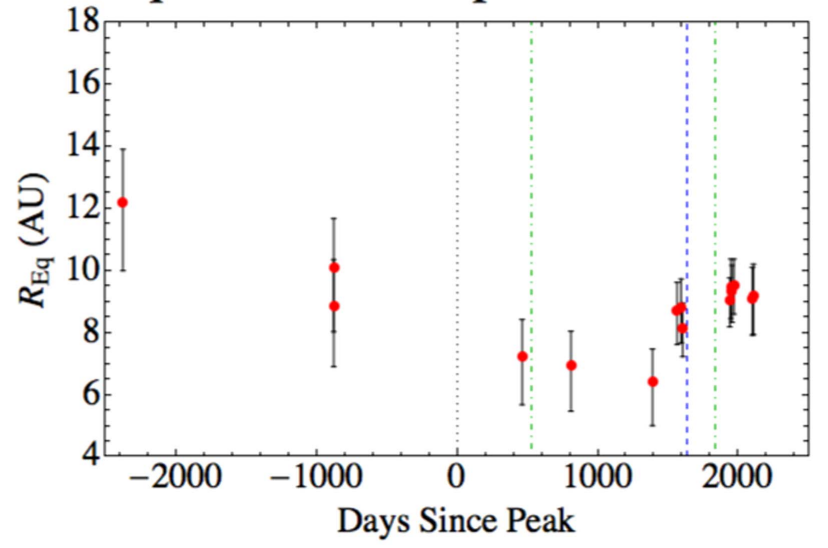

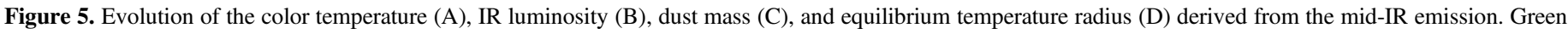
dotted-dashed lines and blue dashed lines correspond to observed re-brightening peaks in optical and X-ray emission, respectively.

Luminosities of the optical counterpart to SN 2010da were estimated by integrating over the temperature-fitted blackbodies. The highest measured luminosity is $3 \times 10^{6} L_{\odot}$ and is consistent with the initial optical brightness peak. After the peak, the luminosity rapidly decreased by over two orders of magnitude to $\sim 4 \times 10^{4} L_{\odot}$ at the end of the early epoch $\left(t_{d}=101\right.$ days). At the intermediate-time re-brightening on day $\sim 530$, the luminosity peaked at $\sim 1.4 \times 10^{5} L_{\odot}$ and decreased by a factor of approximately two in the following 40 days. In the late epoch, the optical counterpart exhibited luminosities ranging from $\sim 5-10 \times 10^{4} L_{\odot}$, which is consistent with the luminosity minimum of the intermediate epoch.

\subsection{Progenitor and Post-outburst DUSTY SED Models}

Spectral energy distributions (SEDs) from multi-wavelength photometry of SN 2010da and its progenitor can be modeled with the radiative transfer code DUSTY (Ivezic \& Elitzur 1997) to provide additional constraints the properties of the emitting dust and heating source before and after the outburst. DUSTY models are fitted to flux measurements taken on day -2379 and between days 1602 and $1641,{ }^{16}$ which are referred to as the progenitor and post-outburst models, respectively. Models were fit by minimizing the reduced $\chi^{2}$ statistic. For both

\footnotetext{
${ }^{16}$ gri and $\mathrm{JHK}_{\mathrm{S}}$ observations were taken on days 1636.7 and 1641.1, respectively, and the closest 3.6 and $4.5 \mu \mathrm{m}$ observations were made on day 1602 .
}

models, dust is assumed to be in a spherical shell with a radial density profile proportional to $r^{-2}$ and made up of amorphous carbon with an MRN (Mathis et al. 1977) grain size distribution $\left(a_{\min }=0.005 \mu \mathrm{m}, a_{\max }=0.25 \mu \mathrm{m}\right.$, and agrain size power law proportional to $a^{-3.5}$ ). Based on the average of the measured temperatures of the optical counterpart (Figure 6(A)), a $7000 \mathrm{~K}$ blackbody is adopted as the central heating source for both models. The impact on the model fitting is negligible when assuming temperatures within the range of 6000-8000 K.

The free parameters are the heating source luminosity $\left(L_{*}\right)$, optical depth through the dust shell $\left(\tau_{V}\right)$, shell thickness as a multiplicative factor, $Y$, and the dust temperature at the inner radius $\left(T_{\mathrm{in}}\right)$. Note that the inner radius size, $r_{\mathrm{in}}$, is determined from DUSTY by $L_{*}$ and $T_{\mathrm{in}}$. Following the analysis of Khan et al. (2015), the fitted parameters from DUSTY can be used to estimate the total mass of the shell, $M_{\mathrm{e}}$, as follows.

$$
M_{\mathrm{e}}=\frac{4 \pi r_{\mathrm{in}}^{2} \tau_{V}}{\kappa_{V}},
$$

where $\kappa_{V}$ is the V-band opacity of the dust, which is assumed to be $100 \mathrm{~cm}^{2} \mathrm{~g}^{-1}$. Due to the unresolved nature of the emission from SN 2010da, there is a lack of morphological information which limits the utility of the DUSTY models. $Y$ is therefore not well constrained by the mid-IR fluxes, especially since it will not trace cooler, extended dust

It is important to note that the true morphology of the dust in the system may not resemble the spherical geometry assumed 


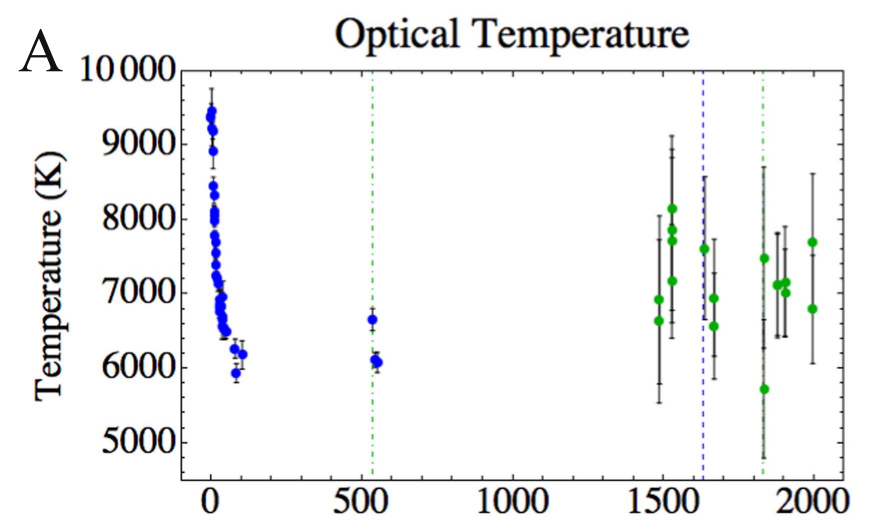

B

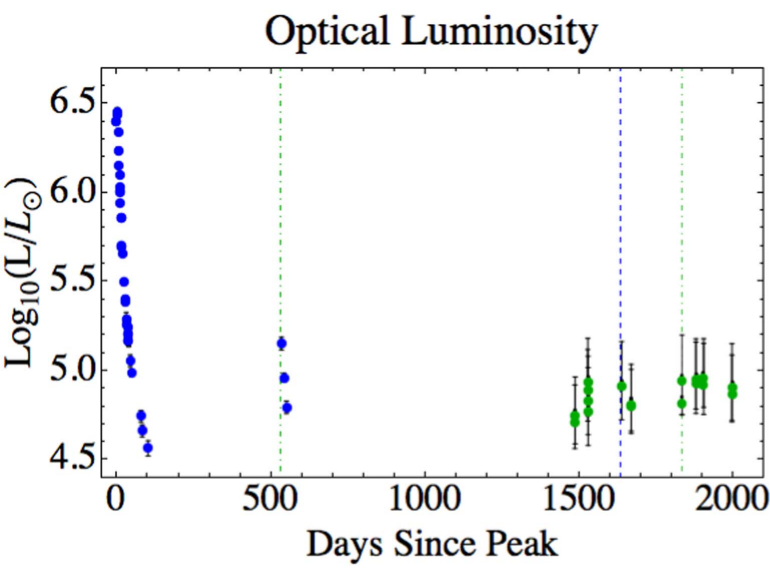

Figure 6. Temperature (A) and luminosity (B) of the optical counterpart determined from $B V I$ (blue points) and gi (green points) photometry. $R$ - and $r$ band photometry were neglected in the temperature and luminosity estimates due to possible contamination from $\mathrm{H} \alpha$ line emission. Green dotted-dashed lines and blue dashed lines correspond to observed re-brightening peaks in optical and X-ray emission, respectively.

for the DUSTY models and likely differs before and after the initial outburst. Additionally, due to the lack of far-IR photometry, the luminosity and optical depth derived by DUSTY assume that there is only a single distribution of hot dust traced by the mid-IR emission. This derived heating source luminosity, however, is well-defined since the observed optical to mid-IR flux measurements and limits provide robust constraints on the shape and amplitude of the warm dust SED. The parameters and constraints derived from the DUSTY models are shown in Table 4.

The progenitor model shown in Figure 7(A) indicates the lower limits of $T_{\text {in }}$ and $\tau_{V}$, and the upper limit of $r_{\text {in }}$. Constraints on the optical depth $\left(\tau_{V} \gtrsim 5\right)$ were determined based on the deep $r$ - and $i$-band limits for the optical component. There is a degeneracy between $T_{\text {in }}$ and $\tau_{V}$ since enhanced near/mid-IR emission from hotter dust can be mitigated by increasing optical depth. A grid search was performed over the free parameters $T_{\text {in }}, Y$, and $\tau_{V}>5$, which revealed lower limits for $T_{\text {in }}$ and $Y$ of $1000 \mathrm{~K}$ and $1,{ }^{17}$ respectively. The luminosity of the heating source is $L_{*}=1.5 \pm 0.2 \times 10^{4} L_{\odot}$, consistent with the integrated IR luminosity inferred from the analysis in Section 3.2.1 (Figure 5(B)).

The best-fit model for the post-outburst SED (Figure 7(B)) was determined by a grid search over $T_{\mathrm{in}}, Y$, and $\tau_{V}$. A search

\footnotetext{
${ }^{17} Y=1$ is the lowest allowable value from DUSTY.
}

through a range of values for the shell thickness of $Y=2,10$, 20, 100, and 1000 indicated that the SED models are poorly constrained for $Y \gtrsim 10$. Assuming $Y=100$, the best-fit parameters for $T_{\text {in }}$ and $\tau_{V}$ are $1700 \pm 200 \mathrm{~K}$ and $0.7 \pm 0.1$, respectively. Model fits indicate that the post-outburst luminosity is almost an order of magnitude more $\left(L_{*}=\right.$ $\left.1.0 \pm 0.2 \times 10^{5} L_{\odot}\right)$ than the progenitor and is consistent with the estimated late-time optical luminosity (Figure 4(B)). Additionally, the best-fit optical depth reflects the positive detections of the post-outburst optical counterpart, which exhibits less local extinction than the optically thick progenitor. Excess emission at the $r$-band compared to the $g$ - and $i$-band fluxes provides evidence for strong $\mathrm{H} \alpha$ line emission. The model SED in the near-IR is slightly steeper and lower than the apparent slope and flux of the $\mathrm{JHK}_{s}$ measurements. This may be due to dust temperature changes that occurred within the $\sim 40$ days between the optical/nearIR and mid-IR observations as well as the greater uncertainty associated with the near-IR photometry.

Both progenitor and post-outburst models indicate similar limits on the inner dust radii of $\sim 3-6 \times 10^{14} \mathrm{~cm}(\sim 20-40 \mathrm{au})$. The estimated equilibrium temperature radii at days -2379 and 1590 are less than $\sim 1 / 2$ of $r_{\text {in }}$ (Figure 5(D)); however, this is not unexpected since the equilibrium temperature radius is determined from the temperature derived by the 3.6 and $4.5 \mu \mathrm{m}$ flux from the entire shell, whereas $r_{\text {in }}$ corresponds to the hottest dust at its inner edge. The upper limit of the total shell mass in the progenitor model, $M_{\mathrm{e}}<12 \times 10^{-5} M_{\odot}$, bounds the mid-IR derived dust mass assuming a gas-to-dust ratio of 100 (see Table 3). The shell mass derived from the post-outburst model, $M_{\mathrm{e}}=0.4 \pm 0.1 \times 10^{-5} M_{\odot}$, is a factor of approximately two lower than the mass implied from the mid-IR analysis, which may be attributed deviations from the spherical morphology, grain size distribution, and/or $r^{-2}$ radial density profile assumed for the models.

\section{DISCUSSION}

\subsection{Mid-IR Dust Evolution of SN 2010da}

\subsubsection{Evaporation from the Optical Flash}

The significant decrease in reddening and optical depth toward SN 2010da during and after the outburst (Bond 2010; Brown 2010) imply the destruction of the circumstellar dust that heavily enshrouded the progenitor. The decrease in the derived mass between the progenitor and post-outburst DUSTY models (Table 4) is suggestive of dust destruction as well. Given the luminosity of the optical flash from the initial outburst $\left(L \approx 3 \times 10^{6} L_{\odot}\right)$, we can estimate the radius, $r_{\text {sub }}$, out to where the optical/UV flux will evaporate dust by heating it beyond the sublimation temperature $\left(T_{\text {sub }} \sim 1700 \mathrm{~K}\right.$; e.g., Gall et al. 2011). The sublimation radius can be estimated from Equation (2) to be $r_{\text {sub }} \approx 4 \times 10^{14} \mathrm{~cm}(\sim 27 \mathrm{au})$. Since $r_{\text {sub }}$ is greater than the pre-outburst equilibrium temperature radius shown in Figure 5(D), we conclude that a significant amount of circumstellar dust was evaporated in the initial optical flash. The lower post-outburst dust mass estimates at early and intermediate times relative to the progenitor dust mass despite higher post-outburst luminosities strengthens this claim. 
Table 4

DUSTY Model Parameters and Constraints on Circumstellar Dust

\begin{tabular}{|c|c|c|c|c|c|c|}
\hline Epoch & $T_{\text {in }}(\mathrm{K})$ & $L_{*}\left(\times 10^{4} L_{\odot}\right)$ & $\tau_{V}$ & $Y$ & $r_{\text {in }}\left(\times 10^{14} \mathrm{~cm}\right)$ & $\overline{M_{e}\left(\times 10^{-5} M_{\odot}\right)}$ \\
\hline Progenitor & $\gtrsim 1000$ & $1.5 \pm 0.2$ & $\gtrsim 5$ & $\gtrsim 1$ & $\lesssim 6.1$ & $\lesssim 12$ \\
\hline Post-Outburst & $1700 \pm 200$ & $10 \pm 2$ & $0.7 \pm 0.1$ & $100^{\mathrm{a}}$ & $2.9 \pm 0.7$ & $0.4 \pm 0.1$ \\
\hline
\end{tabular}

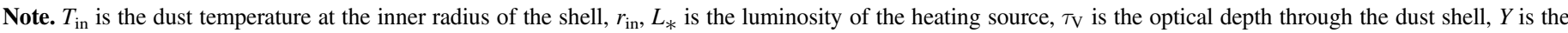
geometrical thickness as a multiplicative factor of $r_{\mathrm{in}}$, and $M_{\mathrm{e}}$ is the total mass of the shell derived from Equation (3).

${ }^{\mathrm{a}} Y$ is fixed at a value of 100 for the post-outburst model.

\section{A SN2010da Progenitor - Nov 2003}

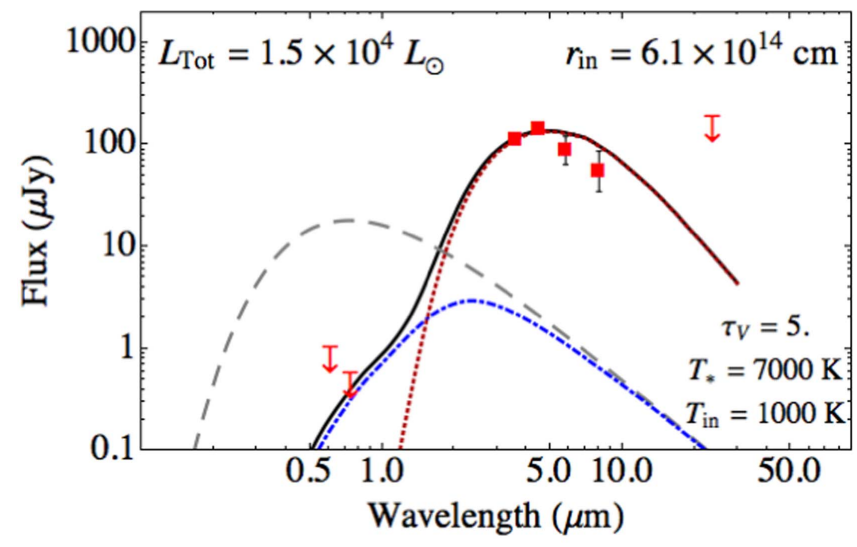

\section{B SN2010da After Outburst - Late 2014}

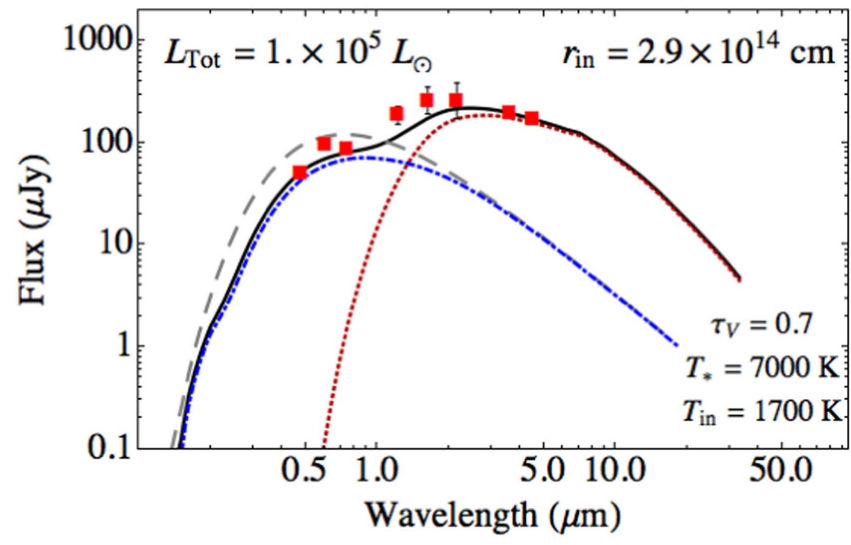

Figure 7. DUSTY radiative transfer models of the SN 2010da progenitor (A) and after the outburst (B). The gray dashed line represents the unabsorbed spectrum from the heating source. The blue dotted-dashed and red dotteddashed lines correspond to emission from attenuated stellar heating source and dust emission, respectively. The black solid line indicates the total spectra outputted from the system.

\subsubsection{Post-outburst Re-brightening: Evidence of \\ Progenitor Survival and On-going Dust Formation}

The prominent post-outburst re-brightening across IR, optical, and X-ray wavelengths at around day $\sim 1600$ (Figure 3) implicates the survival of the progenitor. However, we must consider the alternative explanation where the re-brightening arises from interactions between a shock driven by the initial outburst with shells or clumps of circumstellar material. We claim that this is unlikely due to the hardness variability of the post-outburst X-ray emission reported by Binder et al. (2016): high luminosity measurements taken on days 120 and 1635 exhibit harder X-ray emission than the low-luminosity emission observed on day 1450 (see Figure 3(C)). The hardening and increasing X-ray energetics between days 1450 and 1635 are indicative of continued outburst activity from a surviving central system. Such variability is difficult to reconcile with a terminal outburst. Given the apparent survival of the progenitor, we propose that the late-time (days $\sim 1500-2100$ ) re-brightening of the IR luminosity by over a factor of two times the progenitor (Figure 5(B)) is due to an increase in the luminosity of the central system and on-going dust formation.

At intermediate times (days 460-1390), the dust temperature exhibits a similar increasing trend as the IR luminosity, whereas the late-time temperature shows a sudden drop while the luminosity continues to increase until day $\sim 2000$. The intermediate-time temperature-luminosity correlation is consistent with the scenario where a fixed quantity of emitting dust is at a constant distance (i.e., the dust formation radius) from a heating source increasing in brightness: $T_{\mathrm{d}} \propto L^{1 / 4}$ (Equation (2)). Constant dust masses estimated at intermediate times (Figures 5(C) and (D)) strengthen this interpretation. Ultimately, we are not sensitive to changes in the luminosity from possible warm and/or cold dust distributions and therefore require far-IR photometry to conclusively claim that the heating source is increasing in luminosity. However, it is apparent from the evolution of the midIR dust properties and variability of the temperature and luminosity of the optical counterpart (Figure 6) that the energetics of the system is significantly changing many years after the initial eruption.

Mid-IR emission observed after the outburst must be associated with dust that either survived the initial optical/ UV flash, reformed in the supersaturated vapor of the previously evaporated grains, and/or reformed in the outflow from the central source. Alternatively, the emitting dust could be interstellar in nature and illuminated by the optical flash (i.e., an "IR echo"). We first test the IR echo scenario by using Equation (2) to estimate the predicted temperature of dust at a distance of $c t / 2$, which is where the hottest dust is located if heated by the optical/UV flash (e.g., Fox et al. 2011, 2015). At $t \approx 460$ days and given an optical/UV outburst luminosity of $L \approx 3 \times 10^{6} L_{\odot}$, the predicted dust temperature is $43 \mathrm{~K}$, significantly less than the temperature estimated at day 460 $\left(T_{\mathrm{d}} \approx 850 \pm 73 \mathrm{~K}\right)$. It is therefore unlikely that the postoutburst mid-IR emission is due to an IR echo.

The unchanging equilibrium temperature radius of the emitting dust at intermediate times ( $\sim 8$ au, Figure 5(D)) over $\sim 2.5$ years suggests that the mid-IR emission is not associated with dust reformation in the progenitor winds nor survival from the optical/UV flash. Dust formed through either channel would unlikely be located at radii that are smaller than the radii estimated for the progenitor dust, especially given an approximate shock velocity of $\sim 660 \mathrm{~km} \mathrm{~s}^{-1}$ based on the FWHM of the $\mathrm{H} \alpha$ emission line observed from SN 2010da 
during the outburst (Chornock \& Berger 2010). Given a shock velocity of $660 \mathrm{~km} \mathrm{~s}^{-1}$, the shock that would sweep up reformed or surviving dust (e.g., Kochanek 2011) will propagate $\sim 300$ au over 2.5 year and is over an order of magnitude larger than the estimated dust radius. Although we cannot rule out the presence of surviving or reformed cold dust due to the lack of far-IR measurements, we claim that the postoutburst emission in the mid-IR is primarily associated with newly formed dust from the surviving central system.

\subsection{On the Nature of SN 2010da}

The heavily dust-enshrouded nature of the progenitor, and the low peak visual luminosity compared to core-collapse $\mathrm{SNe}$, seem at first to suggest that SN 2010da was an intermediateluminosity transient similar to NGC 300 OT2008-1 (Bond et al. 2009; Humphreys et al. 2011) and SN 2008S (Thompson et al. 2009; Kochanek 2011). However, the post-outburst evolution of SN 2010da is significantly different from those of SN 2008S and NGC 300 OT2008-1, both of which are prototypes of this class of transient. One of the most prevalent interpretations reported in ATels is that SN 2010da is an outburst from a dust-enshrouded LBV (e.g., Chornock \& Berger 2010; Elias-Rosa et al. 2010; Khan et al. 2010). A confounding factor in the SN 2008S-like transient and LBV eruption interpretations is the high $0.3-10 \mathrm{keV}$ X-ray luminosity of $L_{x} \sim 4.5 \times 10^{38} \mathrm{erg} \mathrm{s}^{-1}$ detected within hours of the optical detection (Immler et al. 2010) and recurring, less luminous $\left(L_{x} \sim 4 \times 10^{37} \mathrm{erg} \mathrm{s}^{-1} ; 0.35-8 \mathrm{keV}\right)$ outbursts (Binder et al. 2016). Based on the high X-ray emission and pre-outburst IR luminosity, Binder et al. (2016) argue that the system is an HMXB where the optical companion is an LBV. We instead propose that the optical companion of the HMXB system is a supergiant ( $\mathrm{sg}) \mathrm{B}[\mathrm{e}]$ star.

We discuss possible interpretations for SN 2010da given the new insight from observations of its long-term mid-IR and optical evolution. We consider the following (possibly overlapping) hypotheses for the nature of SN 2010da: an SN 2008S-like transient, an outburst from an $\mathrm{LBV} / \mathrm{sgB}[\mathrm{e}]-$ HMXB, a presumed stellar merger event like V1309 Sco (Tylenda et al. 2011), V838 Mon (Bond et al. 2003, Afşar \& Bond 2007) or the recently identified NGC 4490-OT (Smith et al. 2016), or a low-luminosity supernova explosion (e.g., electron-capture).

\subsubsection{Comparison with SN 2008S-like Events and Low-luminosity SNe}

The growth in IR luminosity from SN 2010da directly contrasts with the late-time mid-IR light curves of SN 2008 S and OT2008-1 that decrease to over a magnitude below the mid-IR flux from the respective progenitors (Figure 8). Notably, SN 2008S and OT2008-1 did not have any detections of X-ray emission, whereas the peak X-ray luminosity from SN 2010da was $L_{x} \sim 4.5 \times 10^{38} \mathrm{erg} \mathrm{s}^{-1}$ (Immler et al. 2010). Subsequent X-ray and optical outbursts like those observed from SN 2010da may be hidden by substantial quantities of circumstellar dust still present around SN 2008S and OT2008$1\left(\tau_{V} \gtrsim 100\right.$ assuming surviving stars with similar luminosity as their progenitor; Adams et al. 2015); however, this high value of $\tau_{V}$ is also in disagreement with the moderate optical depth estimated from the post-outburst SED of SN 2010da $\left(\tau_{V} \sim 1\right.$; Figure $\left.7(\mathrm{~B})\right)$. One may argue that differences in

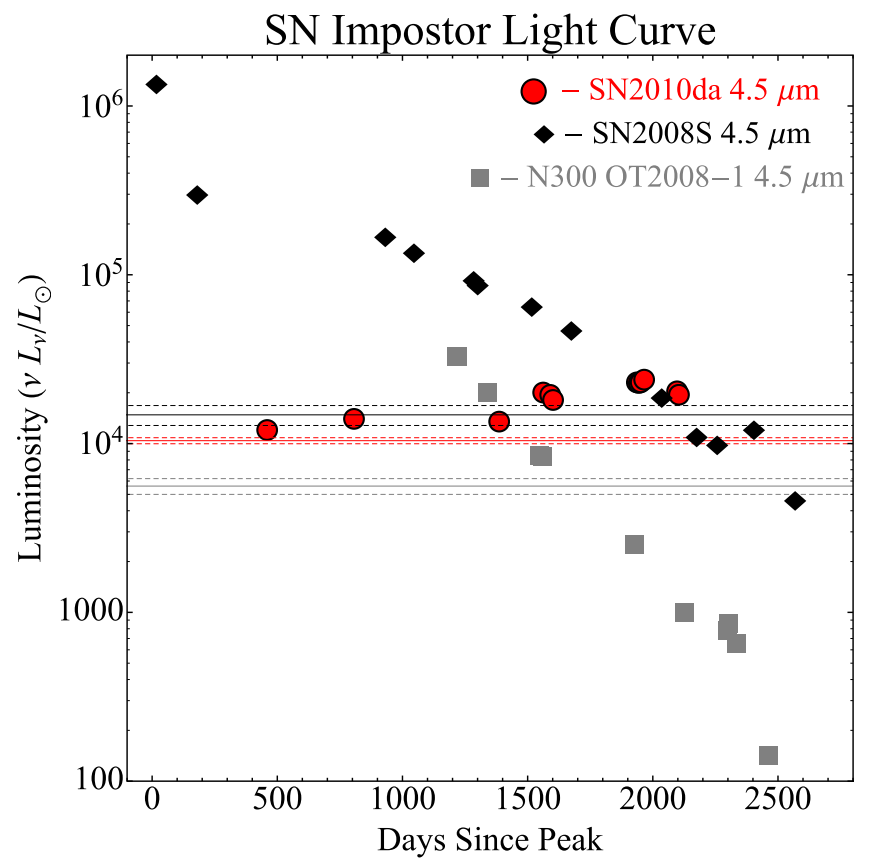

Figure 8. $4.5 \mu \mathrm{m}$ luminosity light curve of SN 2008S and NGC 300 OT2008-1 (Adams et al. 2015) overplotted with SN 2010da. The solid lines correspond to the progenitor flux and the dashed lines correspond to the $1 \sigma$ uncertainty in the measured flux.

viewing angles or dust shell morphology may explain the nondetection of X-ray emission; however, it is difficult to invoke geometry as a means to reconcile the contrasting evolution of the late-time IR luminosity. Given the evidence we presented, we claim that SN 2010da does not fall within the class of SN 2008S-like transients.

Low-luminosity $\mathrm{SNe}$ have been proposed as the mechanism for 2008S-like events and may provide an alternative explanation for the SN 2010da outburst. In this scenario, SN 2010da's late-time increase in luminosity may be due to energy input from the shock driven into the circumstellar material by the initial outburst. Interestingly, the high X-ray luminosity detected from the initial outburst is consistent with the total integrated luminosity of the system at late times (see Figures 7(B) and 8). However, the $\mathrm{SN}$-interpretation is highly unlikely given the re-brightening events at mid-IR, optical, and $\mathrm{X}$-ray wavelengths and the hardness variability of the X-ray emission (Binder et al. 2016) that imply the survival of the progenitor system.

\subsubsection{A Stellar Merger?}

The prospect of SN 2010da corresponding to a merger event is interesting given the increasing attention on stellar mergers (e.g., Smith et al. 2016); however, this interpretation is unlikely due to the SN 2010da's high X-ray luminosity and late-time rebrightening. Additionally, the smoothly declining optical light curve following the initial outburst of SN 2010da (Figure 4) is not consistent with the almost periodic peaks exhibited from merger candidates V838 Mon and NGC 4490 OT.

Interestingly, V838 Mon, the prototypical system believed to be representative of a stellar merger, exhibited a peak absolute $V$-band magnitude of about -9.8 (Sparks et al. 2008), similar to the SN 2010da peak $\left(M_{V} \sim-10.3\right)$. Antonini et al. (2010) report variable X-ray emission from V838 Mon $\sim 6$ with 
luminosities of $\sim 10^{32-33} \mathrm{erg} \mathrm{s}^{-1} \sim 6$ years after the initial outburst that is believed to be a consequence of interaction between its ejecta and early-type companion. It is difficult to reconcile the discrepancy in the $\mathrm{X}$-ray energetics between V838 Mon and SN 2010da given the $\gtrsim 3$ orders of magnitude difference in X-ray luminosity; therefore, we rule out the merger scenario for SN 2010da.

\subsubsection{An $L B V$ or $S g B[e] H M X B$ ?}

The high X-ray luminosity of the initial outburst and subsequent re-brightening present strong evidence for an HMXB interpretation (Binder et al. 2016) since stellar winddriven mechanisms have not been observed to reach such high luminosity. For example, the highest X-ray luminosity from a known LBV is $\sim 10^{34} \mathrm{erg} \mathrm{s}^{-1}$ (Nazé et al. 2012), which is four orders of magnitude less than the peak X-ray luminosity from SN 2010da. Interestingly, optical spectra taken around the initial optical peak reveal emission lines indicative of an LBVlike outburst (e.g., $\mathrm{H} \alpha$, He I, Fe II, and [N II]; Chornock \& Berger 2010; Elias-Rosa et al. 2010). Binder et al. (2016) therefore suggest that SN 2010da is associated with an eruption from an LBV-HMXB system. They substantiate this claim from the $\lesssim 5$ Myr age estimate for the population of nearby stars inferred from the CMD. LBVs, however, are not found to exhibit luminosities lower than $\lesssim 2.5 \times 10^{5} L_{\odot}$ (Smith et al. 2004), which is over a factor of four greater than the progenitor luminosity estimated from the SED model (Figure 7(A)). We propose that the optical companion of the HMXB is instead an sgB[e]-star.

$\mathrm{SgB}[\mathrm{e}]-$ stars resemble LBVs spectroscopically and share a similar region in the HR diagram; however, $\operatorname{sgB}[\mathrm{e}]$-stars can be less luminous than LBVs and do not exhibit giant eruptions. The significant increase of the luminosity during the optical outburst $\left(\sim 3 \times 10^{6} L_{\odot}\right)$ is therefore the strongest evidence against the $\mathrm{sgB}[\mathrm{e}]$ hypothesis. Although individual $\mathrm{sgB}[\mathrm{e}]$ stars do not exhibit significant variability, increases in the luminosity of the optical continuum concurrent with X-ray outbursts have been observed from sgB[e]-HMXB systems. For example, optical observations of the April 1998 X-ray outburst $\left(L_{\mathrm{X}} \sim 3 \times 10^{38} \mathrm{erg} \mathrm{s}^{-1}\right)$ of the galactic sgB[e]-HMXB CI Cam revealed lower limits on the optical outburst amplitude of a factor of two to five times the continuum level of the progenitor $^{18}$ (Hynes et al. 2002). This is still over an order of magnitude less than the amplitude of the initial optical outburst from SN 2010da, but is consistent with the intermediate and late-time re-brightening events (Figure 6). Analogous to CI Cam, the optical variability from SN 2010da is likely due to interactions between the $\operatorname{sgB}[\mathrm{e}]-\mathrm{star}$ and its compact companion.

The process(es) driving the optical outburst in $\mathrm{sgB}[\mathrm{e}]-$ HMXBs can be the heating of the $\mathrm{sgB}[\mathrm{e}]$ star, reprocessing of the X-ray emission, direct emission from ejecta, and/or interaction between ejecta and existing circumstellar material (e.g., Hynes et al. 2002). For SN 2010da, heating of the optical companion is unlikely since the $B-V$ color remains roughly constant following the outburst (Figure 4(D)) and the effective temperature only exhibits a range of $\sim 6000-10,000 \mathrm{~K}$ (Figure 6 (A)). Reprocessing of X-ray emission is also unlikely given the analysis of the archival Swift data by Villar et al. (2016), who show the X-ray emission taken within 40 days of the initial

$\overline{18}$ The peak of the optical outburst was missed in these observations.

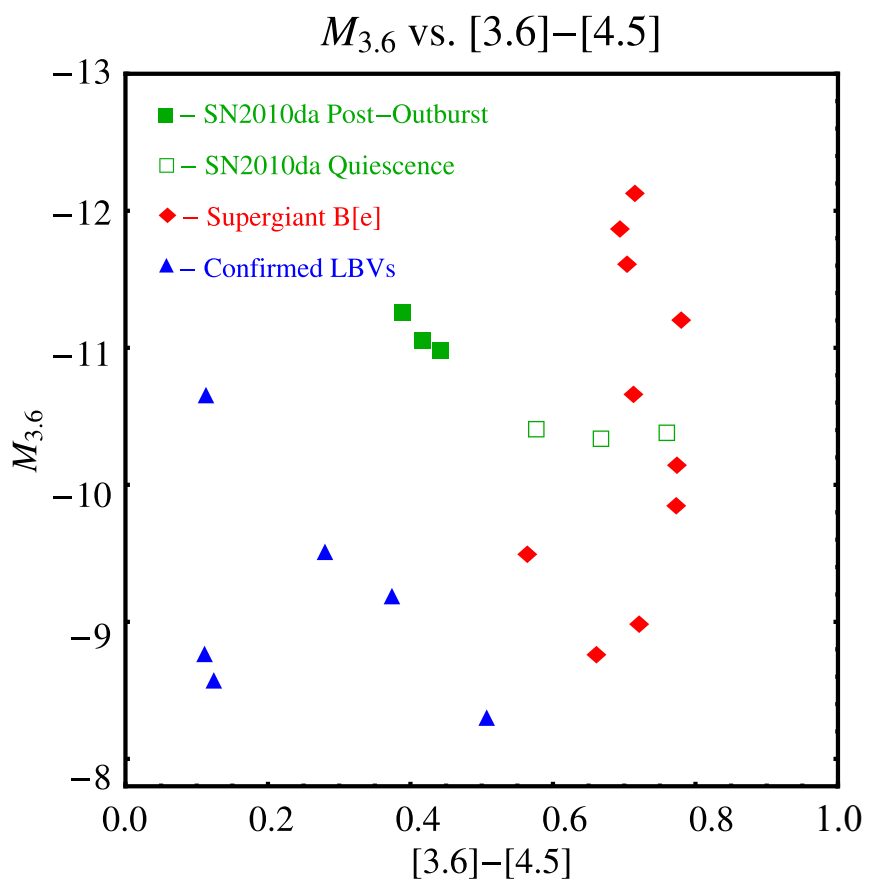

Figure 9. Mid-IR color-magnitude diagram for $\mathrm{sgB}[\mathrm{e}]$ stars and bona-fide LBVs in the Large Magellanic Cloud (Bonanos et al. 2009) overplotted with measurements of SN 2010da in "quiescence" ( $t<0$ day) and post-outburst $(t \sim 2000$ days $)$.

outburst decays at a faster rate than the optical emission. The most plausible explanation for the optical outburst is therefore the direct emission from ejecta and/or interactions of the ejecta with circumstellar material. This is identical to the interpretation of the optical outburst from CI Cam (Hynes et al. 2002).

A comparison of the mid-IR color and magnitudes of known $\mathrm{sgB}[\mathrm{e}]$ stars and bona-fide LBVs in the Large Magellanic Cloud (Bonanos et al. 2009) to the SN 2010da progenitor (in "quiescence") and after the eruption ("post-outburst") are shown in Figure 9. The mid-IR color and absolute magnitude of the progenitor most resemble the $\mathrm{sgB}[\mathrm{e}]$ stars. Similar to the progenitor, the $\mathrm{sgB}[\mathrm{e}]$ stars presented by Bonanos et al. (2009) exhibit mid-IR excesses consistent with hot $\sim 600 \mathrm{~K}$ circumstellar dust. Interestingly, SN 2010da migrated to bluer colors during and following the eruption, which places it in between the $\operatorname{sgB}[\mathrm{e}]$ and $\mathrm{LBV}$ population in the CMD.

Although there are few known, bona-fide sgB[e]-HMXB systems (CI Cam, Hynes et al. 2002; IGR J16318-4848, Filliatre \& Chaty 2004), the observed luminosities and mass in circumstellar material from the literature (Filliatre \& Chaty 2004; Thureau et al. 2009; Chaty \& Rahoui 2012) are similar to what we derive for SN 2010da. From DUSTY models fit to the pre- and post-outburst SED of CI Cam, Thureau et al. (2009) derive a pre-outburst luminosity and dust mass of $2 \times 10^{5} L_{\odot}$ and $3 \times 10^{-6} M_{\odot}$, and a post-outburst luminosity and dust mass of $6 \times 10^{4} L_{\odot}$ and $8 \times 10^{-7} M_{\odot}$. Chaty \& Rahoui (2012) estimate a dust mass of $1.4 \times 10^{-8} M_{\odot}$ by fitting a $T_{\mathrm{d}} \sim 800$ disk model to the near- to mid-IR spectra of IGR J16318-4848 and assuming a distance of $1.6 \mathrm{kpc}$ to the system. The stellar luminosity of the optical companion at a distance of $1.6 \mathrm{kpc}$ is $\sim 6 \times 10^{4} L_{\odot}$. However, Chaty \& Rahoui (2012) mention the system may be further away out to $\sim 4 \mathrm{kpc}$, which would imply a dust mass of $\sim 9 \times 10^{-8} M_{\odot}$ and a luminosity of $\sim 4 \times 10^{5} L_{\odot}$. The late-time optical luminosity 
$\left(\sim 10^{5} L_{\odot}\right)$ and hot dust mass $\left(1.4 \times 10^{-7} M_{\odot}\right)$ we estimate for SN 2010da are therefore comparable to that of the other $\mathrm{sgB}$ [e]-HMXB systems. Notably, infrared spectroscopy of IGR J16318-4848 reveal significant intrinsic optical absorption $\left(A_{V} \sim 6\right.$; Chaty \& Rahoui 2012), which is consistent with the local extinction estimated for the SN 2010da progenitor (Figure 7(A)).

Based on the similar properties to other systems and the midIR color of the progenitor, we argue that SN 2010da is likely an $\mathrm{sgB}[\mathrm{e}]-\mathrm{HMXB}$. Ages of $\mathrm{sgB}[\mathrm{e}]$ stars are also comparable to the $\lesssim 5 \mathrm{Myr}$ age inferred from the CMD of stars in the vicinity of SN 2010da (Binder et al. 2016). However, since there are no confirmed LBV-HMXB systems, there is not enough information to conclusively rule out the LBV-HMXB hypothesis.

It is important to discuss the possible non-supergiant $\mathrm{BeXB}$ interpretation of SN 2010da as proposed by Binder et al. (2011) since they are more common than $\mathrm{sgB}[\mathrm{e}]-\mathrm{HMXBs}$ and also exhibit X-ray outbursts with luminosities exceeding $\sim 10^{38} \mathrm{erg} \mathrm{s}^{-1}$. The most significant difference between $\operatorname{sgB}[\mathrm{e}]$ and Be stars is the lack of hot circumstellar dust around Be stars (e.g., Zickgraf 1999). Given the clear presence of hot dust around SN 2010da and its progenitor, it is highly unlikely to be a non-supergiant Be star.

\subsubsection{Orbital Constraints of the HMXB}

In this section, we assume that $\mathrm{SN} 2010 \mathrm{da}$ is a $\operatorname{sgB}[\mathrm{e}]-$ HMXB system with a neutron star (NS) companion and derive constraints on its orbital parameters based on the observed outburst properties. High X-ray luminosity of the outbursts from $\mathrm{sgB}[\mathrm{e}]-\mathrm{HMXB}$ systems are powered by accretion onto the NS. If the NS accretes material from the $\operatorname{sgB}[\mathrm{e}]$ star's winds, the large amplitude variation of the X-ray luminosity by over two orders of magnitude suggests that the NS is in a highly eccentric orbit. We can then place constraints on the orbital eccentricity, $e$, period, $P_{\text {orb}}$, and semimajor axis, $a$, of the system given the measurements of the varying X-ray luminosity. We note that the X-ray emission may also be due to mass transfer via RLOF; however, X-ray emission from HMXBs is typically dominated by accretion from stellar winds. Additionally, $\operatorname{sgB}[\mathrm{e}]$ stars are not known to exhibit significant changes in radius given their unvarying spectral profile.

Under the assumption of Bondi-Hoyle accretion, the X-ray luminosity from the NS in a HMXB can be expressed as

$$
L_{\mathrm{X}}=\eta \dot{M}_{\mathrm{acc}} c^{2},
$$

where $\eta$ is a constant that depends on the physics of the accretion for which we adopt a value of $\sim 0.1$ (Oskinova et al. 2012), $\dot{M}_{\text {acc }}$ is the mass accretion rate of the NS, and $c$ is the speed of light. For accretion fed by stellar winds of massloss rate $\dot{M}_{\mathrm{w}}$ and velocity $v_{\mathrm{w}}$ regulated by the orbital motion of the NS, $\dot{M}_{\text {acc }}$ is

$$
\dot{M}_{\mathrm{acc}}=4 \pi \xi \frac{\left(G M_{\mathrm{NS}}\right)^{2}}{v_{\mathrm{rel}}^{3}} \frac{\dot{M}_{\mathrm{w}}}{4 \pi r^{2} v_{\mathrm{w}}}
$$

where $\xi$ is a factor accounting for radiation pressure and the finite cooling time of the gas for which we assume is $\sim 1$ (e.g., Oskinova et al. 2012), $M_{\mathrm{NS}}$ is the mass of the NS, $v_{\text {rel }}$ is the velocity of the NS relative to the winds, and $r$ is the orbital separation between the stellar wind source and the NS. The relative velocity is simply related to the wind velocity and the orbital velocity of the NS, $v_{\text {orb }}$, as follows.

$$
v_{\text {rel }}=\sqrt{v_{\text {orb }}^{2}+v_{\mathrm{w}}^{2}} \text {. }
$$

Using the vis-viva equation, $v_{\text {orb }}$ can be re-expressed as

$$
v_{\text {orb }}=\sqrt{G M_{*}\left(\frac{2}{r}-\frac{1}{a}\right)},
$$

where $M_{*}$ is the mass of the $\operatorname{sgB}[\mathrm{e}]$ star and $G$ is the gravitational constant.

The eccentricity of the system can be constrained assuming that the observed X-ray luminosity peak, $L_{\mathrm{X}, \mathrm{p}}$, occurs during periapse $(r=a(1-e))$ and that the lowest measured X-ray luminosity, $L_{\mathrm{X}, \mathrm{a}}$, occurs during apoapse $(r=a(1+e))$. The ratio between the peak and minimum measured X-ray luminosity is $\sim 100$ (Binder et al. 2016), which implies $L_{\mathrm{X}, \mathrm{p}} / L_{\mathrm{X}, \mathrm{a}}>100$. It then follows from Equations (4)-(6) that

$$
\frac{L_{\mathrm{X}, \mathrm{p}}}{L_{\mathrm{X}, \mathrm{a}}}=\frac{\left(v_{\mathrm{orb}, \mathrm{a}}^{2}+v_{\mathrm{w}}^{2}\right)^{3 / 2}(1+e)^{2}}{\left(v_{\mathrm{orb}, \mathrm{p}}^{2}+v_{\mathrm{w}}^{2}\right)^{3 / 2}(1-e)^{2}}>100,
$$

where $v_{\mathrm{orb}, \mathrm{a}}$ and $v_{\mathrm{orb}, \mathrm{p}}$ are the orbital velocities of the NS during apoapse and periapse, respectively. We assume that $v_{\text {orb }}<v_{w}$ at apoapse and periapse, which implies

$$
\frac{\left(v_{\mathrm{orb}, \mathrm{a}}^{2}+v_{\mathrm{w}}^{2}\right)^{3 / 2}(1+e)^{2}}{\left(v_{\mathrm{orb}, \mathrm{p}}^{2}+v_{\mathrm{w}}^{2}\right)^{3 / 2}(1-e)^{2}} \approx \frac{(1+e)^{2}}{(1-e)^{2}}>100 .
$$

The lower limit on the eccentricity is therefore

$$
e>0.82 \text {. }
$$

By combining Equations (4)-(7), we can constrain the semimajor axis and orbital period from the limits on the orbital eccentricity from the X-ray luminosity at periapse:

$$
\begin{aligned}
L_{\mathrm{X}, \mathrm{p}} \approx & 0.1 c^{2} \frac{\dot{M}_{\mathrm{w}}}{(a(1-e))^{2} v_{\mathrm{w}}} \\
& \times \frac{\left(G M_{\mathrm{NS}}\right)^{2}}{\left(G M_{*}\left(\frac{2}{a(1-e)}-\frac{1}{a}\right)+v_{\mathrm{w}}^{2}\right)^{3 / 2}} .
\end{aligned}
$$

At the late-time $\mathrm{X}$-ray luminosity peak around day $\sim 1600$, where $L_{\mathrm{X}, \mathrm{p}} \sim 4 \times 10^{37} \mathrm{erg} \mathrm{s}^{-1}$ (Binder et al. 2016), we infer a stellar wind mass-loss rate of $\dot{M}_{\mathrm{w}} \sim 1.4 \times 10^{-5} M_{\odot} \mathrm{yr}^{-1}$ from our analysis in Section 3.2.1 and adopt a gas-to-dust mass ratio of 100 . We assume a wind velocity of $v_{\mathrm{w}} \sim 660 \mathrm{~km} \mathrm{~s}^{-1}$ based on the widths of the emission lines observed during the optical re-brightening at intermediate times (Chornock \& Berger 2010; Elias-Rosa et al. 2010; Chornock et al. 2011). Adopting $M_{*} \sim 40 M_{\odot}$ for the $\operatorname{sgB}[\mathrm{e}]$ star and $M_{\mathrm{NS}} \sim 1.5 M_{\odot}$ for the NS, we can show from Equation (11) that

$$
a \gtrsim 0.26 \text { au and } P_{\text {orb }} \gtrsim 8 \text { days. }
$$

Based on the $\sim 400$ day timespan between the apparent local maxima at days 1562 and 1965 in the late-time mid-IR light curve (see Figure 3(A)), we place upper limits on the orbital period and semimajor axis assuming the IR peaks correspond to the periastron passage of the NS:

$$
P_{\text {orb }} \lesssim 400 \text { days and } a \lesssim 3.6 \text { au. }
$$


Known sgHMXBs typically exhibit orbital periods of 10 days or less with low eccentricities $(e \lesssim 0.3$; Reig 2011) due to short circularization timescales. The long orbital periods and high eccentricities inferred for the system are therefore consistent with the Binder et al. (2016) interpretation of SN 2010da as a newly formed HMXB. Circularization timescales estimated for known sgHMXBs range from $\sim 10^{4}-10^{7}$ years (Stoyanov \& Zamanov 2009), which suggests that the presumed HMXB associated with SN 2010da is less than $10^{4}$ years old.

We can glean insight on the orbital and disk/shell configuration of the system from the upper limit derived for the semimajor axis of the binary. Estimates of the dust shell/ disk radius indicate sizes greater than 7 au (see Figure 5(D)), which implies the disk/shell likely surrounds the entire binary system. Interactions between the $\operatorname{sgB}[\mathrm{e}]$ star and compact companion may, therefore, be linked to the formation of the circumbinary disk/shell. Notably, the rapid increase of the observed mass of hot dust on day 1562 that occurred within $\sim 100$ days of the late-time X-ray outburst (Figure $5(\mathrm{C})$ ) is consistent with this claim. It is plausible that ejecta from the late-time outburst interacted with and swept-up the older dust distribution observed at intermediate times, thereby driving the material out further from the central system (day 1500 in Figure 5(D)). The interaction of ejecta with existing circumstellar material is also consistent with the proposed mechanism driving the optical counterpart of the outburst for this system and CI Cam (Hynes et al. 2002). Additional multi-wavelength and spectroscopic follow-up observations will be required to further substantiate our claim.

\section{CONCLUSIONS}

In this paper, we presented observations of the optical and mid-IR outbursts and recurring re-brightening events of the "impostor" SN 2010da (Figure 1). Both optical and mid-IR light curves reveal re-brightening events in the years following the initial outburst. We attribute the mid-IR emission to hot $(\sim 700-1000 \mathrm{~K})$ dust in the vicinity of SN 2010da and use the 3.6 and $4.5 \mu \mathrm{m}$ flux measured by Spitzer/IRAC to estimate the evolution of the temperature, mass, luminosity, and radius of the emitting dust (Figure 5). Results from this analysis revealed that the IR luminosity of the hot dust increased beyond that of the progenitor as well as a rapid increase in the observed hot dust mass at late times ( 1600 days) after the outburst. We similarly use the optical light curve to study the evolution of the temperature and luminosity of the optical component (Figure 6). These results indicated a peak optical temperature and luminosity of $\sim 9500 \mathrm{~K}$ and $3 \times 10^{6} L_{\odot}$, respectively, during the initial outburst.

At pre- and post-outburst epochs with sufficient multiwavelength photometry, we fit DUSTY models to the SEDs in order to obtain further constraints of the dust and heating source properties. Both progenitor and post-outburst models provide parameters that are in agreement with the properties inferred from the mid-IR and optical light curve analysis. We note that the properties inferred for the models only constrain the mid-IR emitting dust that is not sensitive to colder dust that may exist at further distances from the central system.

We argue that significant quantities of pre-existing dust were destroyed via sublimation from the initial optical flash of SN 2010da by comparing the sublimation radius to the equilibrium temperature radius of the progenitor. This claim is also substantiated by the low post-outburst hot dust mass estimated at early and intermediate times relative to the progenitor dust mass. The enhanced mid-IR emission after the outburst must therefore arise from surviving, pre-existing dust, dust reformed in the winds of the progenitor, and/or newly formed dust from the outflow of the central system. We rule out surviving and reformed dust based on the the disagreement between the predicted temperatures from an IR echo of the initial optical flash and the observed hot dust temperature, and the disagreement between the expected shock radius with derived equilibrium temperature radius. Since the re-brightening emission (Figure 3) and the hardness variability of the $\mathrm{X}$-ray spectrum (Binder et al. 2016) implicate the survival of the progenitor, we claim that the post-outburst mid-IR emission is most likely associated with newly formed dust from the central system.

The nature of SN 2010da presents an interesting mystery since it exhibited an LBV-like eruption, recurring high X-ray luminosity bursts, and re-brightening mid-IR emission. Analysis of the observed properties allowed us to rule out interpretations of SN 2010da as a 2008S-like transient, a merger product, or a low-luminosity supernova. We address the leading theory of SN 2010da as an LBV-HMXB as proposed by Binder et al. (2016) and instead suggest it is an $\operatorname{sgB}[\mathrm{e}]-$ HMXB. Our claim is substantiated by the low progenitor luminosity relative to known LBVs and the shared location of the progenitor in a mid-IR CMD with $\mathrm{sgB}[\mathrm{e}]$ stars in the LMC as cataloged by Bonanos et al. (2009, Figure 9). Additionally, SN 2010da exhibits similar luminosities and dust masses to the two bona-fide $\operatorname{sgB}[\mathrm{e}]-\mathrm{HMXB}$ systems CI Cam and IGR J16318-4848. We, however, do not rule out an LBV-HMXB interpretation given the similarities between $\mathrm{sgB}[\mathrm{e}]$ stars and LBVs, and because there are currently no confirmed LBVHMXB systems from which to draw a comparison.

Under the sgB[e]-HMXB interpretation of SN 2010da, we were able to place constraints on its orbital parameters. Given that the X-ray emission from HMXBs typically arises from accretion of stellar winds from the optical companion onto the NS, the variations of the measured X-ray luminosity could be used to constrain the eccentricity, orbital period, and semimajor axis of the system. Our calculations indicate a high eccentricity $(e>.82)$ and long orbital period ( $P_{\text {orb }} \gtrsim 8$ days) relative to known HMXBs, which supports the interpretation of SN 2010da as a newly formed HMXB.

Such a rare system presents unique opportunities to study how the interaction between the companions might influence the mass loss, luminosity, and temperature of the optical component as well as the orbital evolution of the system. Interestingly, the upper limits we placed on the semimajor axis that were determined by the apparent periodicity of mid-IR rebrightening events indicate that the system is surrounded by a circumbinary disk/shell of hot dust. Interactions between the $\mathrm{sgB}[\mathrm{e}]$ and NS at periapse may be linked to the changing properties of the surrounding dust, such as the increase in the observed hot dust mass that occurred around the same time as the recurring X-ray outburst at day $\sim 1600$ (Figure 5(D)). Follow-up observations monitoring the mid-IR, optical, and $\mathrm{X}$-ray light curves will provide invaluable information on the connection between the stellar component, compact object, and circumstellar/binary dust in the system. 
This work made use of observations from the Spitzer Space Telescope operated by the Jet Propulsion Laboratory, California Institute of Technology, under a contract with NASA (PIDS 1083, 40204, 61002, 80015A, 80196, 80015, 10136, 10139, 11063 , and 11053). Ground-based observations presented were obtained from the Bronberg Observatory, the SMARTS Consortium $1.3 \mathrm{~m}$ telescope at CTIO, the $1 \mathrm{~m}$ Swope telescope at LCO, the Mount Lemmon Observing Facility, operated by the University of Minnesota, and the Magellan Baade Telescope at LCO. This work was partially carried out at the Jet Propulsion Laboratory, California Institute of Technology, under a contract with the National Aeronautics and Space Administration. J.J. is supported by the National Science Foundation Graduate Research Fellowship under Grant No. DGE-1144469. R.D.G. and his group at MLOF were supported, in part, by the United States Air Force.

R.L. thanks Drew Clausen for enlightening discussions on high-mass X-ray binaries. R.L. also thanks Scott Adams for insightful comments and the anonymous referee for the valuable suggestions and corrections. H.E.B. thanks the STScI Director's Discretionary Research Fund for supporting STScI's participation in the SMARTS Consortium. We appreciate the excellent work of the CTIO/SMARTS service observers who obtained the ANDICAM images during many long clear Tololo nights: Juan Espinoza, Alberto Miranda, Mauricio Rojas, and Jacqueline Seron.

\section{REFERENCES}

Adams, S. M., Kochanek, C. S., Prieto, J. L., et al. 2015, arXiv:1511.07393 Afşar, M., \& Bond, H. E. 2007, AJ, 133, 387

Antonini, F., Montez, R., Jr., Kastner, J. H., et al. 2010, ApJ, 717, 795

Berger, E., \& Chornock, R. 2010, ATel, 2638

Binder, B., Williams, B. F., Kong, A. K. H., et al. 2011, ApJL, 739, L51

Binder, B., Williams, B. F., Kong, A. K. H., et al. 2016, MNRAS, 457, 1636

Bonanos, A. Z., Massa, D. L., Sewilo, M., et al. 2009, AJ, 138, 1003

Bond, H. E. 2010a, ATel, 2640

Bond, H. E., Bedin, L. R., Bonanos, A. Z., et al. 2009, ApJL, 695, L154

Bond, H. E., Henden, A., Levay, Z. G., et al. 2003, Natur, 422, 405

Brown, P. J. 2010b, ATel, 2633

Chaty, S., \& Rahoui, F. 2012, ApJ, 751, 150

Chaty, S., Rahoui, F., Foellmi, C., et al. 2008, A\&A, 484, 783

Chornock, R., \& Berger, E. 2010, ATel, 2637

Chornock, R., Czekala, I., \& Berger, E. 2011, ATel, 3726

Coleiro, A., Chaty, S., Zurita Heras, J. A., Rahoui, F., \& Tomsick, J. A. 2013, A\&A, 560, A108

Compiègne, M., Flagey, N., Noriega-Crespo, A., et al. 2010, ApJL, 724, L44
Contreras, C., Hamuy, M., Phillips, M. M., et al. 2010, AJ, 139, 519

Dalcanton, J. J., Williams, B. F., Seth, A. C., et al. 2009, ApJS, 183, 67

Draine, B. T., \& Li, A. 2007, ApJ, 657, 810

Dwek, E. 1987, ApJ, 322, 812

Dwek, E., Arendt, R. G., Bouchet, P., et al. 2008, ApJ, 676, 1029

Elias-Rosa, N., Mauerhan, J. C., \& van Dyk, S. D. 2010, ATel, 2636, 1

Fazio, G. G., Hora, J. L., Allen, L. E., et al. 2004, ApJS, 154, 10

Filliatre, P., \& Chaty, S. 2004, ApJ, 616, 469

Fox, O. D., Chevalier, R. A., Skrutskie, M. F., et al. 2011, ApJ, 741, 7

Fox, O. D., Silverman, J. M., Filippenko, A. V., et al. 2015, MNRAS, 447, 772

Gall, C., Hjorth, J., \& Andersen, A. C. 2011, A\&ARv, 19, 43

Gehrz, R. D., Roellig, T. L., Werner, M. W., et al. 2007, RScI, 78, 011302

Guerrero, M. A., \& Chu, Y.-H. 2008, ApJS, 177, 216

Hamuy, M., Folatelli, G., Morrell, N. I., et al. 2006, PASP, 118, 2

Humphreys, R. M., Bond, H. E., Bedin, L. R., et al. 2011, ApJ, 743, 118

Hynes, R. I., Clark, J. S., Barsukova, E. A., et al. 2002, A\&A, 392, 991

Immler, S., Brown, P., \& Russell, B. R. 2010, ATel, 2639

Ivezic, Z., \& Elitzur, M. 1997, MNRAS, 287, 799

Khan, R., Kochanek, C. S., Stanek, K. Z., \& Gerke, J. 2015, ApJ, 799, 187

Khan, R., Stanek, K. Z., Kochanek, C. S., Thompson, T. A., \& Prieto, J. L. 2010, ATel, 2632

Kochanek, C. S. 2011, ApJ, 741, 37

Landolt, A. U. 1992, AJ, 104, 372

Laskar, T., Berger, E., \& Chornock, R. 2010, ATel, 2648, 1

Liu, Q. Z., van Paradijs, J., \& van den Heuvel, E. P. J. 2005, A\&A, 442, 1135

Liu, Q. Z., van Paradijs, J., \& van den Heuvel, E. P. J. 2006, A\&A, 455, 1165

Low, F. J., Rieke, G. H., \& Gehrz, R. D. 2007, ARA\&A, 45, 43

Massey, P., McNeill, R. T., Olsen, K. A. G., et al. 2007, AJ, 134, 2474

Mathis, J. S., Rumpl, W., \& Nordsieck, K. H. 1977, ApJ, 217, 425

Milligan, S., Cranton, B. W., \& Skrutskie, M. F. 1996, Proc. SPIE, 2863, 2

Monard, L. A. G. 2010, CBET, 2289, 1

Nazé, Y., Rauw, G., \& Hutsemékers, D. 2012, A\&A, 538, A47

Oskinova, L. M., Gayley, K. G., Hamann, W.-R., et al. 2012, ApJL, 747, L25

Persson, S. E., Murphy, D. C., Smee, S., et al. 2013, PASP, 125, 654

Prieto, J. L., Bond, H. E., Kochanek, C. S., et al. 2010, ATel, 2660, 1

Reig, P. 2011, Ap\&SS, 332, 1

Skrutskie, M. F., Cutri, R. M., Stiening, R., et al. 2006, AJ, 131, 1163

Smith, N., Andrews, J. E., Van Dyk, S. D., et al. 2016, MNRAS, 458, 950

Smith, N., Li, W., Silverman, J. M., Ganeshalingam, M., \& Filippenko, A. V. 2011, MNRAS, 415, 773

Smith, N., Vink, J. S., \& de Koter, A. 2004, ApJ, 615, 475

Sparks, W. B., Bond, H. E., Cracraft, M., et al. 2008, AJ, 135, 605

Stoyanov, K. A., \& Zamanov, R. K. 2009, AN, 330, 727

Thompson, T. A., Prieto, J. L., Stanek, K. Z., et al. 2009, ApJ, 705, 1364

Thureau, N. D., Monnier, J. D., Traub, W. A., et al. 2009, MNRAS, 398, 1309

Tinyanont, S., Kasliwal, M. M., Fox, O. D., et al. 2016, arXiv:1601.03440

Tylenda, R., Hajduk, M., Kamiński, T., et al. 2011, A\&A, 528, A114

Van Dyk, S. D., \& Matheson, T. 2012, ApJ, 746, 179

Villar, V. A., Berger, E., Chornock, R., et al. 2016, arXiv:1605.07245

Werner, M. W., Roellig, T. L., Low, F. J., et al. 2004, ApJS, 154, 1

Zickgraf, F.-J. 1999, in IAU Coll. 169, Variable and Non-spherical Stellar Winds in Luminous Hot Stars, ed. B. Wolf, O. Stahl, \& A. W. Fullerton (Berlin: Springer-Verlag), 40

Zubko, V., Dwek, E., \& Arendt, R. G. 2004, ApJS, 152, 211 